\title{
Expansion for moments of regression quantiles with applications to nonparametric testing
}

\author{
ENNO MAMMEN ${ }^{1}$, INGRID VAN KEILEGOM ${ }^{2}$ and KYUSANG Y U 3 \\ ${ }^{1}$ Institut für Angewandte Mathematik, Universität Heidelberg, Im Neuenheimer Feld 205, 69120 Heidel- \\ berg, Germany.E-mail: mammen@math.uni-heidelberg.de \\ ${ }^{2}$ ORSTAT, KU Leuven, Naamsestraat 69, 3000 Leuven, Belgium. E-mail: ingrid.vankeilegom@kuleuven.be \\ ${ }^{3}$ Department of Applied Statistics, Konkuk University, Seoul 143-701, Korea. \\ E-mail: kyusangu@konkuk.ac.kr
}

We discuss nonparametric tests for parametric specifications of regression quantiles. The test is based on the comparison of parametric and nonparametric fits of these quantiles. The nonparametric fit is a NadarayaWatson quantile smoothing estimator.

An asymptotic treatment of the test statistic requires the development of new mathematical arguments. An approach that makes only use of plugging in a Bahadur expansion of the nonparametric estimator is not satisfactory. It requires too strong conditions on the dimension and the choice of the bandwidth.

Our alternative mathematical approach requires the calculation of moments of Nadaraya-Watson quantile regression estimators. This calculation is done by application of higher order Edgeworth expansions.

Keywords: Bahadur expansions; goodness-of-fit tests; kernel smoothing; nonparametric regression; nonparametric testing; quantiles

\section{Introduction}

Consider a data set of $n$ i.i.d. tuples $\left(X_{i}, Y_{i}\right)$, where $Y_{i}$ is a one-dimensional response variable and $X_{i}$ is a $d$-dimensional covariate. For $0<\alpha<1$, we denote the conditional $\alpha$-quantile of $Y_{i}$ given $X_{i}=x$ by $m_{\alpha}(x)$. Thus, we can write

$$
Y_{i}=m_{\alpha}\left(X_{i}\right)+\varepsilon_{i, \alpha} \quad(i=1, \ldots, n),
$$

with error variables $\varepsilon_{i, \alpha}$ that fulfill $q_{\alpha}\left(\varepsilon_{i, \alpha} \mid X_{i}\right)=0$. Here, $q_{\alpha}\left(\varepsilon_{i, \alpha} \mid X_{i}\right)$ is the $\alpha$-quantile of the conditional distribution of $\varepsilon_{i, \alpha}$ given $X_{i}$. Consider the null hypothesis

$$
H_{0}: \text { For all } \alpha \in A \text { there exists a } \theta(\alpha) \in \Theta \text {, such that } m_{\alpha}=m_{\alpha, \theta(\alpha)} \text {, }
$$

where $\left\{m_{\alpha, \theta}: \theta \in \Theta\right\}$ is a parametric class of regression quantiles, $\Theta$ is a compact subset of $\mathbb{R}^{k}$ and $A \subset(0,1)$. The set $A$ can be a singleton $A=\{\alpha\}$, but can also be a closed subset of $(0,1)$ if a set of quantile functions is checked.

In this paper, we aim at studying a test statistic for $H_{0}$, and to study its asymptotic properties under the null and the alternative. We will see that this problem is an example of a quantile model 
where the asymptotics cannot be developed by standard tools of quantile regression. In particular, a direct application of Bahadur expansions requires assumptions that are too restrictive.

Our test statistic is based on kernel smoothing. Let $K\left(u_{1}, \ldots, u_{d}\right)=\prod_{j=1}^{d} k\left(u_{j}\right)$, where $k$ is a one-dimensional density function defined on $[-1,1]$, and let $h=\left(h_{1}, \ldots, h_{d}\right)$ be a $d$ dimensional bandwidth parameter. We assume that all bandwidths $h_{1}, \ldots, h_{d}$ are of the same order. For simplicity of notation, we further assume that they are identical and by abuse of notation we write $h=h_{1}=\cdots=h_{d}$. For any $0<\alpha<1$ and any $x$ in the support $R_{X}$ of $X$, let $F_{\varepsilon_{\alpha} \mid X}(\cdot \mid x)$ be the conditional distribution function of $\varepsilon_{\alpha}=Y-m_{\alpha}(X)$, given $X=x$, and let $r_{\alpha, \theta(\alpha)}(x)$ be the $\alpha$-quantile of $Y-m_{\alpha, \theta(\alpha)}(X)$ given that $X=x$. Define

$$
\widehat{r}_{\alpha}(x)=\arg \min _{r} \sum_{i=1}^{n} K\left(\frac{x-X_{i}}{h}\right) \tau_{\alpha}\left(Y_{i}-m_{\alpha, \widehat{\theta}(\alpha)}\left(X_{i}\right)-r\right),
$$

where $\tau_{\alpha}(u)=\alpha u_{+}-(1-\alpha) u_{-}, u_{+}=u I(u>0)$ and $u_{-}=u I(u<0)$ and where $\widehat{\theta}(\alpha)$ is an estimator of $\theta(\alpha)$.

Note that instead of estimating the conditional quantile $r_{\alpha, \theta(\alpha)}(x)$ by the above estimator, we could have considered the alternative estimator

$$
\widehat{r}_{\alpha}^{\text {alt }}(x)=\arg \min _{m} \sum_{i=1}^{n} K\left(\frac{x-X_{i}}{h}\right) \tau_{\alpha}\left(Y_{i}-m\right)-m_{\alpha, \widehat{\theta}(\alpha)}(x) .
$$

However, the latter estimator has the important drawback that the consideration of responses $Y_{i}$ in a neighborhood of $x$ induces a smoothing bias, whereas $\widehat{r}_{\alpha}(x)$ has no smoothing related bias, since it is based on the errors $Y_{i}-m_{\alpha, \theta(\alpha)}\left(X_{i}\right)$, whose conditional quantile of order $\alpha$ is exactly zero under $H_{0}$ for all $X_{i}$.

We suppose that $A$ is a closed subinterval of $(0,1)$. We define the following test statistic:

$$
\widehat{T}_{A}=\int_{A} \int_{R_{X}} \widehat{r}_{\alpha}(x)^{2} w(x, \alpha) d x d \alpha
$$

for some weight function $w(x, \alpha)$. For the case that $A$ contains only one value $\alpha$ we use

$$
\widehat{T}_{\alpha}=\int_{R_{X}} \widehat{r}_{\alpha}(x)^{2} w(x) d x
$$

for some weight function $w(x)$. One could also generalize our results to the case that $A$ is a finite set that contains more than one element. To keep notation simple we omit this case in our mathematical analysis.

Our test is an omnibus test that has power against all types of alternatives. It is based on the comparison of a kernel quantile estimator with the parametric fit. We will show that the test statistic is asymptotically equivalent to a weighted $L_{2}$-distance between the nonparametric and the parametric estimator. Similar tests have been used in a series of papers for mean regression. Early references are Härdle and Mammen [19], González Manteiga and Cao [15], Hjellvik, Yao and Tjøstheim [23], Zheng [44] and Fan, Zhang and Zhang [13]. Furthermore recent references are Dette and Spreckelsen [10], Kreiss, Neumann and Yao [34], Haag [18], Leucht [36], Gao and 
Hong [14] and Aït-Sahalia, Fan and Peng [1]. Most of the more recent work concentrates on time series data.

The classical way to carry over results from parametric and nonparametric mean regression to quantile regression is the use of Bahadur expansions. The main point is that asymptotically quantile regression is equivalent to weighted mean regression. This approach has been used in Chaudhuri [6], Truong [40], He and $\mathrm{Ng}$ [20], He, $\mathrm{Ng}$ and Portnoy [21] and more recently in Hoderlein and Mammen [24], Hong [25], Kong, Linton and Xia [33], Lee and Lee [35], El Ghouch and Van Keilegom [11], Li and Racine [37], and De Backer, El Ghouch and Van Keilegom [8], see also the papers Chao, Volgushev and Cheng [5] and Volgushev, Chao and Cheng [42] where refinements of Bahadur expansions have been discussed. A detailed review of quantile regression can be found in the book by Koenker [30]. Testing procedures in quantile regression were considered in Zheng [45], Koenker and Machado [31], Bierens and Ginther [4], Horowitz and Spokoiny [26], Koenker and Xiao [32], and He and Zhu [22], among others. They all considered tests for the parametric form of the quantile function. More recently, Rothe and Wied [38] proposed a test statistic for the hypothesis that the conditional distribution belongs to a certain parametric class. Tests based on quantiles of the errors have also been considered in Su and White [39] in the context of testing conditional independence. Other recent papers are the ones by Volgushev et al. [41] and Conde-Amboage, Sánchez-Sellero and González-Manteiga [7], who considered significance tests in quantile regression and developed a test statistic based on marked empirical processes.

In this paper, we will discuss how results from mean regression carry over to our case. Whereas elsewhere a first attempt could be based on the application of a Bahadur expansion, we will see that in our setting the accuracy of a direct application of Bahadur expansions is too poor. We will shortly explain this here for the testing problem where $A$ contains only one value $\alpha$. Suppose for simplicity at this stage that the parametric model contains only one value $\theta_{0}=\theta_{0}(\alpha)$ and that $\widehat{\theta}=\widehat{\theta}(\alpha)=\theta_{0}$. The Bahadur expansion of $\widehat{r}_{\alpha}(x)$ is given by

$$
\tilde{r}_{\alpha}(x)=-\frac{\sum_{i=1}^{n} K\left(\frac{x-X_{i}}{h}\right)\left\{I\left(\varepsilon_{i, \alpha} \leq 0\right)-\alpha\right\}}{\sum_{i=1}^{n} K\left(\frac{x-X_{i}}{h}\right) f_{\varepsilon_{\alpha} \mid X}\left(0 \mid X_{i}\right)},
$$

where $f_{\varepsilon_{\alpha} \mid X}$ is the conditional density of $\varepsilon_{\alpha}$ given $X$. This gives the following approximation for $\widehat{T}_{\alpha}$ :

$$
\widetilde{T}_{\alpha}=\int_{R_{X}} \tilde{r}_{\alpha}(x)^{2} w(x) d x .
$$

One can show that up to a logarithmic factor $\sup _{x}\left|\widehat{r}_{\alpha}(x)-\widetilde{r}_{\alpha}(x)\right|$ and $\sup _{x}\left|\widehat{r}_{\alpha}(x)\right|$ are of order $\left(n h^{d}\right)^{-3 / 4}$ and $\left(n h^{d}\right)^{-1 / 2}$, respectively. This implies that up to a logarithmic factor, the difference $\widehat{T}_{\alpha}-\widetilde{T}_{\alpha}$ is of order $\left(n h^{d}\right)^{-5 / 4}$. On the other hand as it is also the case in mean regression $\widetilde{T}_{\alpha}$ is equal to the sum of a deterministic term and a random term of order $n^{-1} h^{-d / 2}$. Thus, the above approximation only helps if $\left(n h^{d}\right)^{-5 / 4} \ll n^{-1} h^{-d / 2}$ or equivalently if $n h^{3 d} \rightarrow \infty$ for sample size $n$ going to $\infty$. For example, if one applies a bandwidth $h \sim n^{-1 /(4+d)}$ that leads to rate optimal estimation of twice differentiable functions this assumption would allow only a onedimensional setting $d=1$. Also in the case of minimax optimal testing with twice differentiable functions under the alternative (see Ingster [27-29] and Guerre and Lavergne [16]), the optimal bandwidth $h \sim n^{-2 /(8+d)}$ is only allowed for dimension $d=1$. In this paper, we develop an 
asymptotic theory for $L_{2}$-type quantile tests that works under the assumption that $n h^{3 d / 2} \rightarrow \infty$. In the above examples, this allows dimensions $d \leq 7$ and $d \leq 3$. Furthermore, for our asymptotic discussion of the distribution of the test statistic on the hypothesis we only need the assumption that $n h^{d} \rightarrow \infty$. Thus on the hypothesis, our basic assumptions coincide with conditions needed for the asymptotics of mean regression. We conjecture that also for the alternative the assumption $n h^{3 d / 2} \rightarrow \infty$ could be weakened but that then the asymptotic mean of the test statistic changes. We will comment on this after the statement of Theorem 2.

In our approach, we will make use of the fact that Bahadur expansions of kernel quantile estimators calculated at two different points are asymptotically independent if they are calculated at points that are such that the supports of the kernels do not overlap. Thus, the variance of an integral over a Bahadur expansion should be of smaller order than the variance of the Bahadur expansion at a fixed point. The main technical difficulty that will come up when applying this idea is the need to calculate moments of the kernel regression quantiles. We will introduce a method for the expansion of such moments that is based on Edgeworth expansions in a related problem. Our main result gives a bound between the moments of kernel regression quantiles and the moments of its Bahadur approximation.

The paper is organized as follows. In the next section, we will state our result on moments of kernel regression quantiles. Our main result on the asymptotics of $L_{2}$-type quantile tests is given in Section 3. We will also introduce some kind of wild bootstrap procedure adapted to quantile regression and give a theoretical result on its consistency. In Section 4, we present the results of a simulation study, and we analyze data on Engel curves. The proofs are postponed to the last three sections.

\section{Asymptotic moments}

In this section, we will present an asymptotic result on higher order moments of kernel regression quantiles. This result will be our most important ingredient for getting our result on the asymptotic distribution of our test statistic. In our result the moments of kernel regression quantiles are compared with the moments of their Bahadur approximations. Recall that we are interested in the null hypothesis $H_{0}$ defined in (2). We suppose that for all $\alpha \in A$,

$$
m_{\alpha}(\cdot)=m_{\alpha, \theta_{0}(\alpha)}(\cdot)+n^{-1 / 2} h^{-d / 4} \Delta_{\alpha}(\cdot) .
$$

For the case $\Delta_{\alpha} \equiv 0$ the function $m_{\alpha}$ lies on the hypothesis. In order to develop our asymptotic theory, we need to work under the following assumptions. In the formulation of the assumptions and in the proofs, we use the convention that $C, C_{1}, C_{2}, \ldots$ are generic strictly positive constants that are chosen large enough, that $c, c_{1}, c_{2}, \ldots$ are generic strictly positive constants that are chosen small enough, and that $C^{*}, C_{1}^{*}, C_{2}^{*}, \ldots$ are generic strictly positive constants that are arbitrarily chosen. Using this convention, we write $L_{n}=(\log n)^{C}$ for a sequence with $C>0$ large enough and $L_{n}^{*}=(\log n)^{C^{*}}$ for a sequence with an arbitrarily chosen constant $C^{*}>0$. All these variable names are used for different constants and sequences, even in the same equation.

We will make use of the following assumptions.

(B1) The support $R_{X}$ of $X$ is a compact convex subset of $\mathbb{R}^{d}$. The density $f_{X}$ of $X$ is bounded and bounded away from zero on $R_{X}$. The function $\Delta_{\alpha}$ is uniformly absolutely bounded for $\alpha \in A$. 
(B2) The conditional distribution of $\varepsilon_{\alpha}$ given $X=x$ allows a density $f_{\varepsilon_{\alpha} \mid X}(e \mid x)$ that is twice differentiable with respect to $e$. For this derivative it holds that $\left|f_{\varepsilon_{\alpha} \mid X}^{\prime \prime}(e \mid x)\right| \leq C$ for $|e| \leq c, x \in R_{X}$, and $\alpha \in A$. The density $f_{\varepsilon_{\alpha} \mid X}(e \mid x)$ also satisfies $f_{\varepsilon_{\alpha} \mid X}(e \mid x)>0$ and $\left|f_{\varepsilon_{\alpha} \mid X}\left(e^{\prime} \mid x^{\prime}\right)-f_{\varepsilon_{\alpha} \mid X}(e \mid x)\right| \leq C\left(\left\|x^{\prime}-x\right\|+\left|e^{\prime}-e\right|\right)$ for $x, x^{\prime} \in R_{X}$ and $e, e^{\prime} \in \mathbb{R}$, where $\|\cdot\|$ is the Euclidean norm. Moreover, the functions $f_{X}(x), m_{\alpha}(x)$ and $\Delta_{\alpha}(x)$ are continuously differentiable with respect to $x$.

(B3) The bandwidth $h$ satisfies $h=o(1)$ and $n h^{d} / L_{n}^{*} \rightarrow \infty$. The kernel $k$ is a symmetric, continuously differentiable probability density function with compact support, $[-1,1]$, say. It fulfills a Lipschitz condition and it is monotone strictly increasing on $[-1,0]$. It holds that $k^{\prime}\left(k^{-1}(u)\right) \geq \min c\left\{u^{\kappa},(k(0)-u)^{\kappa}\right\}$ for some $0 \leq \kappa<1$ where $k^{-1}:[0, k(0)] \rightarrow$ $[-1,0]$ denotes the inverse of $k:[-1,0] \rightarrow[0, k(0)]$.

In our asymptotics, the density $f_{X}$ and the functions $\Delta_{\alpha}$ are fixed and do not depend on $n$. The cumulative distribution function $F(\cdot \mid x)$ of $Y$ given $X=x$ may depend on $n$. We do not indicate this in our notation.

Assumptions (B1)-(B3) are standard assumptions for the study of smoothing estimators, with the exception of the last assumption in (B3). We now shortly explain why this assumption is needed here. For fixed $u$ and $x=\left(x_{1}, \ldots, x_{d}\right)^{\top}$, define the random vector $V_{n}=$ $\sum_{j=1}^{n}\left(k\left(\frac{x_{1}-X_{1, j}}{h}\right), \ldots, k\left(\frac{x_{d}-X_{d, j}}{h}\right)\right)^{\top}\left\{I\left(\varepsilon_{j, \alpha}^{\Delta} \leq \Delta_{\alpha}^{h}(x)+u\left(n h^{d}\right)^{-1 / 2}\right)-\alpha\right\}$ with $\varepsilon_{j, \alpha}^{\Delta}=\varepsilon_{j, \alpha}+$ $n^{-1 / 2} h^{-d / 4} \Delta_{\alpha}\left(X_{j}\right)$, and where $\Delta_{\alpha}^{h}(x)$ is defined in (10) below. In the proof of the following Theorem 1 we will develop Edgeworth expansions for the distribution of $V_{n}$. Typically, the summands of $V_{n}$ do not fulfill non-lattice type assumptions that are needed for the verification of Edgeworth expansions. But under (B3) a non-lattice assumption can be verified for the conditional distribution of a finite sum of summands of $V_{n}$. For more details, we refer to the proof of Theorem 1. The last assumption in (B3) can be easily verified. It just puts a simple bound on the derivative of $k^{-1}$. For example, it can be easily checked for the triangle kernel and for all kernels of the form $k(z)=\mathbf{1}(|z| \leq 1) c_{r}\left(1-z^{2}\right)^{r}$ with $r \geq 1$. In case that $k^{\prime}$ is bounded away from zero on bounded intervals of $(-1,0)$ the assumption follows if for some $l, l^{*} \in \mathbb{N}$, it holds that $k^{\prime}(x)=(x+1)^{l}+o\left((x+1)^{l}\right)$ for $x \geq-1$ and $x+1$ small enough and that $k^{\prime}(x)=-x^{2 l^{*}+1}+o\left(x^{2 l^{*}}\right)$ for $x$ in a neighborhood of 0 .

We put

$$
\begin{aligned}
& \widehat{r}_{\alpha}^{\Delta}(x)=\arg \min _{r} \sum_{i=1}^{n} K\left(\frac{x-X_{i}}{h}\right) \tau_{\alpha}\left(\varepsilon_{i . \alpha}^{\Delta}-r\right), \\
& \bar{r}_{\alpha}^{\Delta}(x)= \begin{cases}\widehat{r}_{\alpha}^{\Delta}(x) & \text { if }\left|\widehat{r}_{\alpha}^{\Delta}(x)\right| \leq L_{n}\left(n h^{d}\right)^{-1 / 2}, \\
0 & \text { otherwise. }\end{cases}
\end{aligned}
$$

In the main result of this section, we will consider conditional moments of the truncated kernel smoothing quantiles $\bar{r}_{\alpha}^{\Delta}$, conditioned on the number of covariables falling into local neighborhoods. Note that, with positive probability, kernel smoothing quantiles are not defined because there is no covariable in the support of the kernel, with positive probability. Thus unconditional moments are not defined. In the following theorem, we will condition on local neighborhoods $\mathcal{N}^{-}(x)=\left\{u: x_{j}-h \leq u_{j} \leq x_{j}+h\right.$ for all $\left.j=1, \ldots, d\right\}$ that are designed such that the result 
can be easily used for the asymptotic analysis of our test statistic in the next section. Note that $\mathcal{N}^{-}(x)$ is the support of the kernel $h^{-d} K\left(h^{-1}[x-\cdot]\right)$. The theorem could also easily be stated with other local neighborhoods. The conditional moments of the truncated kernel smoothing quantiles $\bar{r}_{\alpha}^{\Delta}$ will be compared with the conditional moments of the following modified Bahadur expansion, denoted by $\widetilde{r}_{\alpha}^{\Delta}(x)$ :

$$
\widetilde{r}_{\alpha}^{\Delta}(x)=\widetilde{r}_{\alpha}^{\Delta,-}(x)+\Delta_{\alpha}^{h}(x),
$$

where $\Delta_{\alpha}^{h}(x)$ is defined such that

$$
E_{x}^{j}\left[K\left(\frac{x-X_{j}}{h}\right)\left\{I\left(\varepsilon_{j, \alpha}^{\Delta} \leq \Delta_{\alpha}^{h}(x)\right)-\alpha\right\}\right]=0 .
$$

Here $E_{x}^{j}$ denotes the conditional expectation, given that $j \in \mathcal{N}^{-}(x)$. Furthermore, $\widetilde{r}_{\alpha}^{\Delta,-}(x)$ is defined as

$$
\begin{aligned}
\tilde{r}_{\alpha}^{\Delta,-}(x) & =-\frac{\sum_{i=1}^{n} K\left(\frac{x-X_{i}}{h}\right)\left\{I\left(\varepsilon_{i, \alpha}^{\Delta} \leq \Delta_{\alpha}^{h}(x)\right)-\alpha\right\}}{\sum_{i=1}^{n} K\left(\frac{x-X_{i}}{h}\right) f_{\varepsilon_{\alpha} \mid X}\left(\Delta_{\alpha}^{h}\left(x, X_{i}\right) \mid X_{i}\right)} \\
& =-\frac{\sum_{i=1}^{n} K\left(\frac{x-X_{i}}{h}\right)\left\{I\left(\varepsilon_{i, \alpha} \leq \Delta_{\alpha}^{h}\left(x, X_{i}\right)\right)-\alpha\right\}}{\sum_{i=1}^{n} K\left(\frac{x-X_{i}}{h}\right) f_{\varepsilon_{\alpha} \mid X}\left(\Delta_{\alpha}^{h}\left(x, X_{i}\right) \mid X_{i}\right)}
\end{aligned}
$$

where

$$
\Delta_{\alpha}^{h}\left(x, X_{j}\right)=\Delta_{\alpha}^{h}(x)-n^{-1 / 2} h^{-d / 4} \Delta_{\alpha}\left(X_{j}\right) .
$$

We have the following asymptotic result for the moments of kernel quantile estimators and their Bahadur approximations.

Theorem 1. Assume (B1)-(B3). Then, for natural numbers $l \geq 1$,

$$
\begin{aligned}
E\left\{\bar{r}_{\alpha}^{\Delta,-}(x)^{2 l}-\widetilde{r}_{\alpha}^{\Delta,-}(x)^{2 l} \mid N^{-}(x)=m\right\} & =O\left(L_{n}\left(n h^{d}\right)^{-l-1}\right), \\
E\left\{\bar{r}_{\alpha}^{\Delta,-}(x)^{2 l-1}-\widetilde{r}_{\alpha}^{\Delta,-}(x)^{2 l-1} \mid N^{-}(x)=m\right\} & =O\left(L_{n}\left(n h^{d}\right)^{-l}\right)
\end{aligned}
$$

uniformly in $x \in R_{X}, \alpha \in A$ and $C_{1}^{*} n h^{d} \leq m \leq C_{2}^{*} n h^{d}$ where $N^{-}(x)$ is the random number of $X_{i}$ 's that lie in $\mathcal{N}^{-}(x)$, and where $\bar{r}_{\alpha}^{\Delta,-}(x)=\bar{r}_{\alpha}^{\Delta}(x)-\Delta_{\alpha}^{h}(x)$. For the second moments of the uncentered estimators $\bar{r}_{\alpha}^{\Delta}$ and $\widetilde{r}_{\alpha}^{\Delta}$ we have that

$$
E\left\{\bar{r}_{\alpha}^{\Delta}(x)^{2}-\widetilde{r}_{\alpha}^{\Delta}(x)^{2} \mid N^{-}(x)=m\right\}=O\left(L_{n} n^{-3 / 2} h^{-5 d / 4}\right) .
$$

Under the additional assumption that $\Delta_{\alpha} \equiv 0$, we get that

$$
E\left\{\bar{r}_{\alpha}^{\Delta}(x)^{2}-\widetilde{r}_{\alpha}^{\Delta}(x)^{2} \mid N^{-}(x)=m\right\}=O\left(L_{n}\left(n h^{d}\right)^{-2}\right) .
$$

We can apply the theorem when $\Delta_{\alpha} \equiv 0$, in which case $\Delta_{\alpha}^{h} \equiv 0$ and $\widetilde{r}_{\alpha}^{\Delta}(x)=\widetilde{r}_{\alpha}^{\Delta,-}(x)$ and $\bar{r}_{\alpha}^{\Delta}(x)=\bar{r}_{\alpha}^{\Delta,-}(x)$. Hence, (11) and (12) hold with $\bar{r}_{\alpha}^{\Delta,-}(x)$ and $\widetilde{r}_{\alpha}^{\Delta,-}(x)$ replaced by $\bar{r}_{\alpha}^{\Delta}(x)$ and $\widetilde{r}_{\alpha}^{\Delta}(x)$. In particular, for $l=1$ (14) follows directly from (11). 


\section{Asymptotic theory}

We suppose that there exists an estimator $\widehat{\theta}(\alpha)$ that converges to $\theta_{0}(\alpha)$. Hence, on the hypothesis the true value of $\theta(\alpha)$ is equal to $\theta_{0}(\alpha)$. On the alternative, $\theta_{0}(\alpha)$ may depend on the chosen estimator $\widehat{\theta}(\alpha)$.

In order to develop the asymptotic distribution of $\widehat{T}_{A}$ and $\widehat{T}_{\alpha}$, we need the following additional assumptions.

(B4) We assume that

$$
\sup _{x \in R_{X}, \alpha \in A}\left|m_{\alpha, \widehat{\theta}(\alpha)}(x)-m_{\alpha, \theta_{0}(\alpha)}(x)-\left(\widehat{\theta}(\alpha)-\theta_{0}(\alpha)\right)^{\top} \gamma_{\alpha}(x)\right|=O_{P}\left(n^{-\frac{1}{2}-c}\right)
$$

for some function $\gamma_{\alpha}(x)$. The function $w(x)$ is continuous, and the functions $w(x, \alpha)$, $\gamma_{\alpha}(x)$ and $\Delta_{\alpha}(x)$ are continuous with respect to $(\alpha, x)$. For $g(x)=w(x, \alpha)$ and $g(x)=$ $w(x)$, it holds that $\left|g\left(x^{\prime}\right)-g(x)\right| \leq C\left\|x^{\prime}-x\right\|$, and $\left|\gamma_{\alpha}\left(x^{\prime}\right)-\gamma_{\alpha}(x)\right| \leq C\left\|x^{\prime}-x\right\|^{\delta}$ for some $0<\delta<1$ and for all $x, x^{\prime} \in R_{X}$ and all $\alpha \in A$.

(B5) For some $\rho>0$, it holds that

$$
\sup _{\alpha \in A}\left\|\widehat{\theta}(\alpha)-\theta_{0}(\alpha)\right\|=O_{P}\left(\left(n^{-1 / 2} h^{-d / 4}\right) / L_{n}^{*} \wedge\left(h^{-\delta} n^{-\frac{1}{2}-\rho}\right) \wedge\left(n^{-\frac{1}{4}} h^{\frac{d}{4}} / L_{n}^{*}\right)\right) .
$$

The first assumption in (B4) can be shown under smoothness conditions on the relation $\theta \rightarrow$ $m_{\alpha, \theta}(x)$. For the case that $A$ contains only one single element, this assumption in (B4) would directly follow from (B5) and the assumption that $\theta \rightarrow m_{\alpha, \theta}(x)$ has a derivative that is continuous in $x$. Assumption (B5) states that $\widehat{\theta}(\alpha)$ achieves at least a nearly parametric rate. In the case of linear quantile regression (i.e., $m_{\alpha}(X)=\theta(\alpha)^{\top} X$ ), such an assumption has been shown in Angrist, Chernozhukov and Fernández-Val [2]. Note that $\sup _{\alpha \in A}\left\|\widehat{\theta}(\alpha)-\theta_{0}(\alpha)\right\|=O_{P}\left(n^{-1 / 2}\right)$ implies (B5) if $\rho$ is chosen such that $h^{\delta} n^{\rho} \rightarrow 0$. Note that $n^{-\frac{1}{2}}=o\left(n^{-\frac{1}{4}} h^{\frac{d}{4}} / L_{n}^{*}\right)$ because of $n h^{d} / L_{n}^{*} \rightarrow \infty$.

We now state our main result on the asymptotic distribution of our test statistics.

Theorem 2. Assume (B1)-(B5). For the case that $\Delta_{\alpha} \not \equiv 0$, make the additional assumption that $n h^{3 d / 2} / L_{n}^{*} \rightarrow \infty$. Then,

$$
\begin{aligned}
& n h^{d / 2} \widehat{T}_{A}-b_{h, A} \stackrel{d}{\rightarrow} N\left(D_{A}, V_{A}\right), \\
& n h^{d / 2} \widehat{T}_{\alpha}-b_{h, \alpha} \stackrel{d}{\rightarrow} N\left(D_{\alpha}, V_{\alpha}\right),
\end{aligned}
$$

where

$$
\begin{aligned}
D_{A} & =\int_{A} \int_{R_{X}} \Delta_{\alpha}(x)^{2} w(x, \alpha) d x d \alpha \\
b_{h, A} & =h^{-d / 2} K^{(2)}(0) \int_{A} \alpha(1-\alpha) \int_{R_{X}} \frac{w(x, \alpha)}{f_{X}(x) f_{\varepsilon_{\alpha} \mid X}^{2}(0 \mid x)} d x d \alpha,
\end{aligned}
$$




$$
\begin{aligned}
V_{A} & =4 K^{(4)}(0) \int_{\alpha, \beta \in A, \alpha<\beta} \alpha^{2}(1-\beta)^{2} \int_{R_{X}} \frac{w(x, \alpha) w(x, \beta)}{f_{X}^{2}(x) f_{\varepsilon_{\alpha} \mid X}^{4}(0 \mid x)} d x d \alpha d \beta, \\
D_{\alpha} & =\int_{R_{X}} \Delta_{\alpha}(x)^{2} w(x) d x \\
b_{h, \alpha} & =h^{-d / 2} K^{(2)}(0) \alpha(1-\alpha) \int_{R_{X}} \frac{w(x)}{f_{X}(x) f_{\varepsilon_{\alpha} \mid X}^{2}(0 \mid x)} d x, \\
V_{\alpha} & =4 K^{(4)}(0) \alpha^{2}(1-\alpha)^{2} \int_{R_{X}} \frac{w^{2}(x)}{f_{X}^{2}(x) f_{\varepsilon_{\alpha} \mid X}^{4}(0 \mid x)} d x,
\end{aligned}
$$

and where for any $j, K^{(j)}(0)$ denotes the $j$-times convolution product of $K$ at 0 .

In our theorem for the alternative, we make the additional assumption that $n h^{3 d / 2} / L_{n}^{*}$ converges to $\infty$. This assumption is used in the proof for the treatment of the deterministic term $T_{n, 2}$, see Lemma 5. The assumption $n h^{3 d / 2} / L_{n}^{*} \rightarrow \infty$ can be weakened but with another limit for $T_{n, 2}$. This would result in a limit theorem for the test statistic with a mean that differs from $b_{h, \alpha}$. We have added a short discussion of this point after the statement of Lemma 5.

We expect that Theorem 2 cannot be used for an accurate calculation of critical values. The asymptotic normality result of Theorem 2 is based on the fact that kernel smoothers are asymptotically independent if they are calculated at points that differ more than $2 h$. Thus, the convergence is comparable to the convergence of the sum of $h^{-d}$ independent summands. This would motivate a rate of convergence of order $h^{-d / 2}$. As has been suggested for other goodness-of-fit tests in the literature, also here a way out is to use a bootstrap procedure. We will introduce some kind of wild bootstrap for quantiles in which the Bahadur expansion $\widetilde{r}_{\alpha}$ of $\widehat{r}_{\alpha}$ is resampled. For the definition of $\tilde{r}_{\alpha}$ see (5) in Section 2. For the bootstrap, we define

$$
\widetilde{r}_{\alpha}^{*}(x)=-\frac{\sum_{i=1}^{n} K\left(\frac{x-X_{i}}{h}\right)\left\{I\left(U_{i} \leq \alpha\right)-\alpha\right\}}{\sum_{i=1}^{n} K\left(\frac{x-X_{i}}{h}\right) \widehat{f}_{\varepsilon_{\alpha} \mid X}\left(0 \mid X_{i}\right)},
$$

where $\widehat{f}_{\varepsilon_{\alpha} \mid X}$ is an estimator of $f_{\varepsilon_{\alpha} \mid X}$ and $U_{i}$ are independent random variables with uniform distribution on $[0,1]$ that are independent of the sample. The bootstrap test statistics are defined as:

$$
\widehat{T}_{A}^{*}=\int_{A} \int_{R_{X}} \widetilde{r}_{\alpha}^{*}(x)^{2} w(x, \alpha) d x d \alpha
$$

and

$$
\widehat{T}_{\alpha}^{*}=\int_{R_{X}} \widetilde{r}_{\alpha}^{*}(x)^{2} w(x) d x .
$$

For proving the consistency of this bootstrap procedure, we do not specify the choice of the estimator $\widehat{f}_{\varepsilon_{\alpha}} \mid X$ that is used in the construction of the bootstrap procedure. We only assume that the estimator is consistent: 
(B6) It holds that

$$
\sup _{\alpha \in A, x \in R_{X}}\left|\widehat{f_{\varepsilon_{\alpha}} \mid X}(0 \mid x)-f_{\varepsilon_{\alpha} \mid X}(0 \mid x)\right| \rightarrow 0
$$

in probability.

The next theorem shows the consistency of the above bootstrap approach.

Theorem 3. Assume (B1)-(B6). Then,

$$
\begin{gathered}
d_{K}\left(\mathcal{L}^{*}\left(n h^{d / 2} \widehat{T}_{A}^{*}-b_{h, A}\right), N\left(D_{A}, V_{A}\right)\right) \stackrel{p}{\rightarrow} 0, \\
d_{K}\left(\mathcal{L}^{*}\left(n h^{d / 2} \widehat{T}_{\alpha}^{*}-b_{h, \alpha}\right), N\left(D_{\alpha}, V_{\alpha}\right)\right) \stackrel{p}{\rightarrow} 0,
\end{gathered}
$$

where $\mathcal{L}^{*}(\ldots)$ denotes the conditional distribution, given the sample. Furthermore, $d_{K}$ is the Kolmogorov distance, that is, the sup norm of the difference between the corresponding distribution functions.

Theorem 3 remains to hold if we replace (B1)-(B5) by weaker conditions. We do not pursuit this because we need for consistency of bootstrap that both, Theorem 2 and Theorem 3, hold.

\section{Numerical study}

In this section, we present the results of our numerical studies. In our first simulation, we show that a direct application of the Bahadur representation is not accurate enough for studying the approximation of the distribution of our test statistics $\widehat{T}_{\alpha}$ and $\widehat{T}_{A}$. For this purpose, we compare the differences $D_{1}=\int\left|\widehat{r}_{\alpha}(x)^{2}-\widetilde{r}_{\alpha}(x)^{2}\right| w(x) d x$ and $D_{2}=\left|\int \widehat{r}_{\alpha}(x)^{2} w(x) d x-\int \widetilde{r}_{\alpha}(x)^{2} w(x) d x\right|$. Here $D_{1}$ is the integrated difference between the quantile regression and its Bahadur representation and $D_{2}$ is the difference between the test statistic and its approximation based on Bahadur representation. It is clear that $D_{2} \leq D_{1}$. Our point is not that $D_{2}$ is smaller than $D_{1}$ but that the ratio $D_{1} / D_{2}$ is large and that it is decreasing for an increasing bandwidth. This result supports our theory that a direct use of Bahadur expansions only works under very restrictive assumptions on the bandwidth. Table 1 shows the results of $D_{1}$ and $D_{2}$ for the one dimensional case. We also simulated a two dimensional model, whose results are shown in Table 2. In the one dimensional model, we set $Y_{i}=X_{i}+\left(0.5 X_{i}+0.5\right) \epsilon_{i}$, where $\epsilon_{i}$ has a standard normal distribution. This results in the $\alpha$ th quantile function $m_{\alpha}(x)=x+0.5 z_{\alpha}(x+1)$, where $z_{\alpha}$ stands for the $\alpha$ th quantile of the standard normal distribution. For the two dimensional model, we set $Y_{i}=X_{1 i}+X_{2 i}+\left(0.5 X_{1 i}-0.5 X_{2 i}+1\right) \epsilon_{i}$, where $\epsilon_{i}$ has a standard normal distribution and we get the $\alpha$ th quantile function $m_{\alpha}\left(x_{1}, x_{2}\right)=x_{1}+2 x_{2}+0.5 z_{\alpha}\left(x_{1}-x_{2}+2\right)$. For the one dimensional model, we generated $X_{i}$ from the uniform distribution supported on the unit interval $(0,1)$. For the two dimensional model, we generated $\left(X_{1 i}, X_{2 i}\right)$ from a distribution on the unit square $(0,1) \times(0,1)$ which has uniform marginals but where the joint distribution differs from a uniform distribution. This is done to allow for a dependence between the two regressors. We generated random vectors from a bivariate normal distribution 
Table 1. Difference between two approximations: $D_{1}$ is the integrated squared approximation error of a quantile estimator by its Bahadur representation and $D_{2}$ is the approximation error of the test statistic $\widehat{T}_{\alpha}$ by $\widetilde{T}_{\alpha}$

\begin{tabular}{|c|c|c|c|c|c|c|c|}
\hline & & & \multicolumn{5}{|c|}{ Bandwidth } \\
\hline & & & 0.05 & 0.08 & 0.1 & 0.12 & 0.15 \\
\hline \multirow[t]{9}{*}{$n=200$} & $\alpha=0.25$ & $D_{1}$ & 0.195 & 0.116 & 0.091 & 0.077 & 0.059 \\
\hline & & $D_{2}$ & 0.067 & 0.044 & 0.037 & 0.034 & 0.028 \\
\hline & & Ratio & 2.922 & 2.653 & 2.473 & 2.284 & 2.073 \\
\hline & $\alpha=0.5$ & $D_{1}$ & 0.231 & 0.139 & 0.110 & 0.091 & 0.071 \\
\hline & & $D_{2}$ & 0.124 & 0.078 & 0.065 & 0.056 & 0.046 \\
\hline & & Ratio & 1.865 & 1.779 & 1.687 & 1.635 & 1.547 \\
\hline & $\alpha=0.75$ & $D_{1}$ & 0.196 & 0.117 & 0.092 & 0.077 & 0.059 \\
\hline & & $D_{2}$ & 0.065 & 0.044 & 0.037 & 0.033 & 0.029 \\
\hline & & Ratio & 3.028 & 2.696 & 2.481 & 2.305 & 2.070 \\
\hline \multirow[t]{9}{*}{$n=400$} & $\alpha=0.25$ & $D_{1}$ & 0.094 & 0.057 & 0.045 & 0.037 & 0.029 \\
\hline & & $D_{2}$ & 0.033 & 0.022 & 0.019 & 0.017 & 0.014 \\
\hline & & Ratio & 2.899 & 2.536 & 2.338 & 2.170 & 2.027 \\
\hline & $\alpha=0.5$ & $D_{1}$ & 0.109 & 0.068 & 0.054 & 0.045 & 0.036 \\
\hline & & $D_{2}$ & 0.058 & 0.039 & 0.032 & 0.028 & 0.023 \\
\hline & & Ratio & 1.867 & 1.755 & 1.684 & 1.614 & 1.552 \\
\hline & $\alpha=0.75$ & $D_{1}$ & 0.094 & 0.056 & 0.045 & 0.037 & 0.029 \\
\hline & & $D_{2}$ & 0.032 & 0.022 & 0.019 & 0.017 & 0.015 \\
\hline & & Ratio & 2.959 & 2.604 & 2.378 & 2.192 & 1.982 \\
\hline
\end{tabular}

with correlations $\rho=0.2$ and 0.8 and then transformed them with their marginal distribution functions. We generated 400 data sets of size 200 and 400 for each model. For the one dimensional model, we used the bandwidths $h=0.05,0.08,0.1,0.12$ and 0.15 and we used the bandwidths $h=0.125,0.150,0.175$ and 0.200 for the two dimensional model. We used the R package quantreg for fitting quantile functions. From Table 1 and Table 2, one can see that the ratio of $D_{1}$ over $D_{2}$ is large for small bandwidths and decreases as the bandwidth grows. This observation supports our approach for the asymptotic theory. This implies that when we approximate the test statistic it requires less strict assumptions on the bandwidth if we approximate the integrated function rather than when we approximate the quantile function itself.

The second simulation study is conducted to show the validity of our bootstrap procedure. We considered four scenarios:

I. All quantiles are linear:

$$
Y_{i}=m_{0}\left(X_{i}\right)+\sigma_{0}\left(X_{i}\right) \epsilon_{i} .
$$

II. The median is linear and other quantiles are not linear:

$$
Y_{i}=m_{0}\left(X_{i}\right)+\sigma_{1}\left(X_{i}\right) \epsilon_{i} .
$$


Table 2. Difference between two approximations under a two dimensional model: $D_{1}$ is the integrated squared approximation error of a quantile estimator by its Bahadur representation and $D_{2}$ is the approximation error of the test statistic $\widehat{T}_{\alpha}$ by $\widetilde{T}_{\alpha}$. The left panel in the table is the result for $\rho=0.2$ and the right panel shows the result for $\rho=0.8$

\begin{tabular}{|c|c|c|c|c|c|c|c|c|c|c|}
\hline & & & \multicolumn{4}{|c|}{ Weak dependence } & \multicolumn{4}{|c|}{ Strong dependence } \\
\hline & & & \multicolumn{8}{|c|}{ Bandwidths } \\
\hline & & & 0.125 & 0.150 & 0.175 & 0.200 & 0.125 & 0.150 & 0.175 & 0.200 \\
\hline \multirow[t]{9}{*}{$n=200$} & $\alpha=0.25$ & $D_{1}$ & 0.249 & 0.139 & 0.086 & 0.058 & 0.153 & 0.105 & 0.077 & 0.059 \\
\hline & & $D_{2}$ & 0.081 & 0.051 & 0.036 & 0.029 & 0.044 & 0.037 & 0.033 & 0.029 \\
\hline & & Ratio & 3.065 & 2.716 & 2.369 & 1.983 & 3.445 & 2.828 & 2.371 & 2.024 \\
\hline & $\alpha=0.5$ & $D_{1}$ & 0.296 & 0.164 & 0.102 & 0.072 & 0.180 & 0.126 & 0.094 & 0.072 \\
\hline & & $D_{2}$ & 0.142 & 0.087 & 0.061 & 0.048 & 0.089 & 0.069 & 0.056 & 0.048 \\
\hline & & Ratio & 2.093 & 1.884 & 1.667 & 1.500 & 2.021 & 1.832 & 1.659 & 1.504 \\
\hline & $\alpha=0.75$ & $D_{1}$ & 0.247 & 0.138 & 0.085 & 0.058 & 0.153 & 0.106 & 0.077 & 0.059 \\
\hline & & $D_{2}$ & 0.077 & 0.047 & 0.035 & 0.029 & 0.045 & 0.037 & 0.032 & 0.029 \\
\hline & & Ratio & 3.221 & 2.929 & 2.446 & 1.990 & 3.355 & 2.831 & 2.378 & 2.022 \\
\hline \multirow[t]{9}{*}{$n=400$} & $\alpha=0.25$ & $D_{1}$ & 0.249 & 0.139 & 0.086 & 0.058 & 0.074 & 0.051 & 0.038 & 0.029 \\
\hline & & $D_{2}$ & 0.081 & 0.051 & 0.036 & 0.029 & 0.023 & 0.019 & 0.017 & 0.015 \\
\hline & & Ratio & 3.066 & 2.716 & 2.369 & 1.983 & 3.273 & 2.716 & 2.278 & 1.958 \\
\hline & $\alpha=0.5$ & $D_{1}$ & 0.296 & 0.166 & 0.102 & 0.072 & 0.087 & 0.061 & 0.046 & 0.035 \\
\hline & & $D_{2}$ & 0.142 & 0.087 & 0.061 & 0.048 & 0.044 & 0.034 & 0.028 & 0.024 \\
\hline & & Ratio & 2.093 & 1.884 & 1.667 & 1.500 & 1.991 & 1.785 & 1.610 & 1.467 \\
\hline & $\alpha=0.75$ & $D_{1}$ & 0.247 & 0.138 & 0.085 & 0.058 & 0.073 & 0.051 & 0.037 & 0.028 \\
\hline & & $D_{2}$ & 0.077 & 0.047 & 0.035 & 0.029 & 0.022 & 0.019 & 0.017 & 0.015 \\
\hline & & Ratio & 3.221 & 2.929 & 2.446 & 1.990 & 3.274 & 2.666 & 2.230 & 1.899 \\
\hline
\end{tabular}


Scenario I

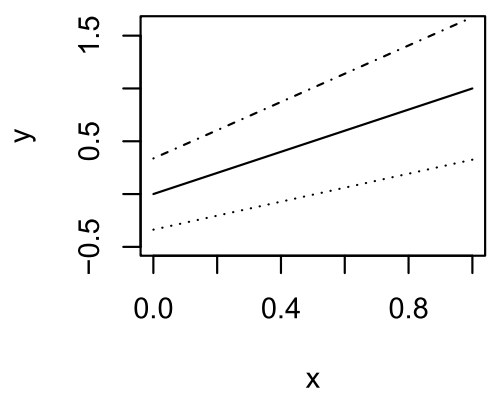

Scenario III-(a)

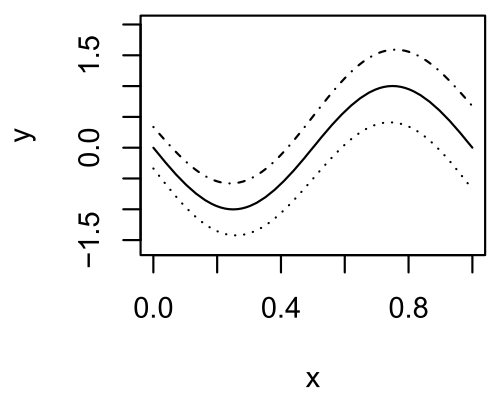

Scenario II

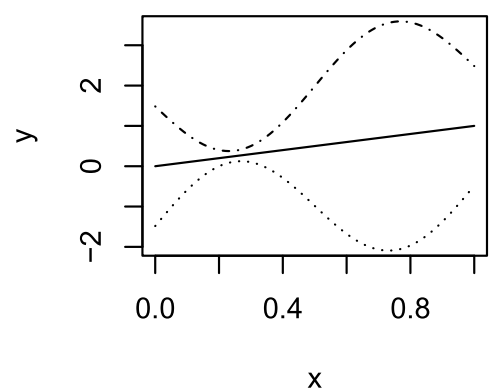

Scenario III-(b)

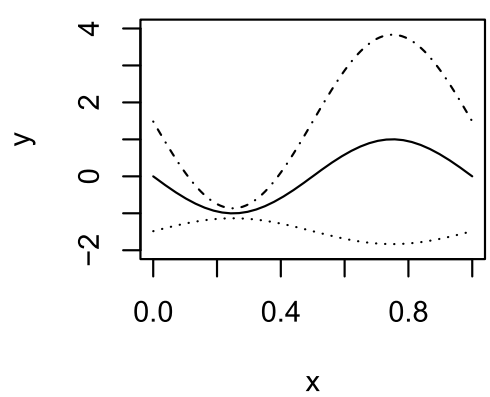

Figure 1. Shape of quantile curves in each scenario. The three curves in each panel represent the $0.25,0.5$, and 0.75 -quantile curves in each scenario.

III. All quantiles are non-linear:
(a) $Y_{i}=m_{1}\left(X_{i}\right)+\sigma_{0}\left(X_{i}\right) \epsilon_{i}$;
(b) $Y_{i}=m_{1}\left(X_{i}\right)+\sigma_{1}\left(X_{i}\right) \epsilon_{i}$.

Here $m_{0}(x)=x, m_{1}(x)=\sin (2 \pi(x-0.5)), \sigma_{0}(x)=\frac{1}{2}(1+x)$, and $\sigma_{1}(x)=2(1.1+$ $\sin (2 \pi(x-0.5)))$. The covariates $X_{i}$ are generated from a uniform distribution on the unit interval $(0,1)$. We generated 200 samples of size 400 . We generated 201 bootstrap samples for each data set. The three scenarios are shown in Figure 1. In the bootstrap procedure, we used a kernel density estimator for estimating the conditional density $f_{\varepsilon_{\alpha} \mid X}(0 \mid x)$.

We tried three bandwidths $0.075,0.100$, and 0.125 for the test statistic, and fifteen choices of bandwidths $(0.1,0.2,0.3) \times(0.050,0.075,0.100,0.125,0.150)$ for estimating the conditional density of the error used in the bootstrap procedure. We applied the proposed bootstrap test for testing the linearity of the lower quartile, the median, and the upper quartile functions. We also tested the linearity hypothesis over these three different quantile levels. In our scenarios, there are four models under the null hypothesis: all three quantiles in scenario I and the median in scenario II. In Table 3, we report the summary statistics of rejection ratios of 45 different choices 
Table 3. First quartile, median and third quartile (obtained from 45 choices of the bandwidth) of the rejection proportions based on 200 generated samples

\begin{tabular}{|c|c|c|c|c|c|}
\hline & & \multicolumn{4}{|c|}{ Quartile functions } \\
\hline & & 1st quartile & Median & 3rd quartile & Sum quartiles \\
\hline \multirow[t]{3}{*}{ Scenario I } & Q1 & 0.005 & 0.000 & 0.005 & 0.000 \\
\hline & Med & 0.010 & 0.005 & 0.010 & 0.005 \\
\hline & Q3 & 0.010 & 0.010 & 0.015 & 0.010 \\
\hline \multirow[t]{3}{*}{ Scenario II } & Q1 & 0.470 & 0.030 & 0.450 & 0.380 \\
\hline & Med & 0.575 & 0.055 & 0.545 & 0.550 \\
\hline & Q3 & 0.630 & 0.105 & 0.610 & 0.695 \\
\hline \multirow[t]{3}{*}{ Scenario III(a) } & Q1 & 0.985 & 1.000 & 0.995 & 1.000 \\
\hline & Med & 1.000 & 1.000 & 1.000 & 1.000 \\
\hline & Q3 & 1.000 & 1.000 & 1.000 & 1.000 \\
\hline \multirow[t]{3}{*}{ Scenario III(b) } & Q1 & 0.065 & 0.300 & 0.735 & 0.545 \\
\hline & Med & 0.135 & 0.385 & 0.865 & 0.770 \\
\hline & Q3 & 0.155 & 0.440 & 0.935 & 0.895 \\
\hline
\end{tabular}

of bandwidths. Among 45 different choices of bandwidths, there was no case where the bootstrap test did not keep the significance level of $5 \%$ under scenario I. In slightly more than half of the cases, the bootstrap test did not keep the significance level in testing the linearity of the median under scenario II. These cases appeared when we used large bandwidths. Concerning the power of the bootstrap test, we observed that almost all choices of the bandwidth showed a power near one under scenario III(a). One exception is the case with the smallest bandwidths where we observe an empirical power around 0.8. One interesting observation is that the bootstrap test shows a poor power for the lower quartile in scenario III(b) where the empirical power ranges from 0.045 to 0.27 . This is however natural since the function is not so far from a linear function as one can see in Figure 1. We also observed that the median and the upper quartile in scenario III(b) showed much stronger power. The result of the test based on the test statistic integrated over levels shows a similar result. In this case, only scenario I is in the null hypothesis and there was no case where the empirical size of the bootstrap test is bigger than 5\%. The power behavior is also similar. The test showed the strongest power with the bigger bandwidths.

Figure 2 shows the distributions of estimated $p$-values by using the proposed bootstrap. The plots are based on 200 simulated data sets for scenario II. The left panel shows the distribution of estimated $p$-values for testing the linearity of the median, which lies in the null and the right panel shows the distribution of estimated $p$-values for testing the linearity of the upper quartile, which lies in the alternative. The distribution in the left panel is close to the uniform distribution which we expect for the null hypothesis and the right panel shows that the bootstrap $p$-values are close to zero, which we also expect.

To summarize our observations from this simulation study, in our setting the bootstrap test keeps the level well except for cases where we use too large bandwidths. On the other hand, too small bandwidths lead to relatively poor power. Interesting cases are scenario II and sce- 


\section{p-value under HO}

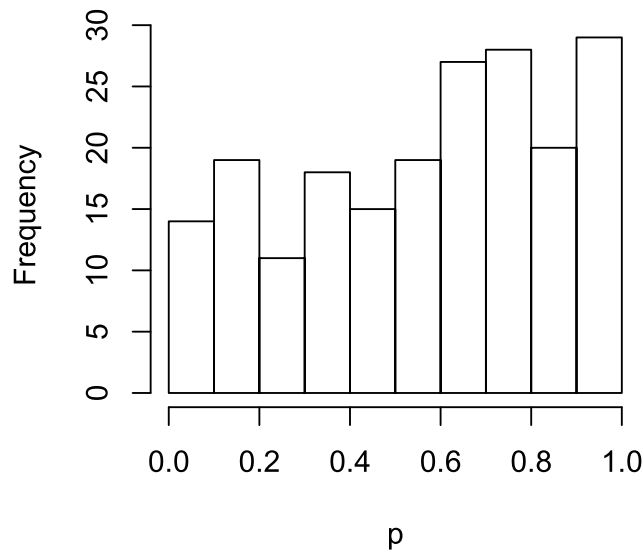

p-value under $\mathrm{H} 1$

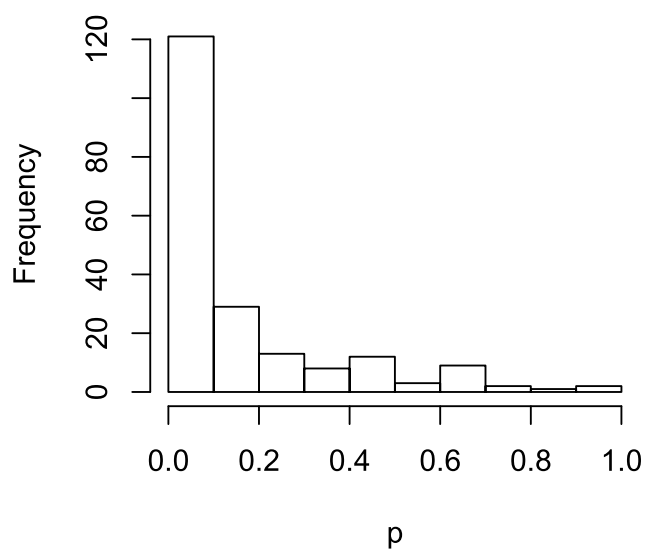

Figure 2. The left panel shows the distribution of estimated $p$-values under the null and the right panel shows the distribution of estimated $p$-values under the alternative.

nario III(b). Under scenario II, the median is linear but both quartiles are not. The simulation result shows that the bootstrap test keeps the level for the median and has some power for the other quantiles. Under scenario III(b), the lower quartile is non-linear but close to the null. We observe that here the bootstrap test has stronger power for the median and the upper quartile than for the lower quartile.

In the last simulation study, we compared our test with the test proposed by Zheng [45]. We considered the same four scenarios as in the previous simulation study. In this simulation, we generated 500 data sets of 400 observations. For the bootstrap, we generated 501 bootstrap samples. The other simulation settings are the same as in the previous simulation study. To choose the bandwidth for Zheng's test, we applied the function npregbw in the R package $n p$, which is based on cross-validation with AIC. For the nonparametric quantile estimator in our procedure, we used the bandwidth proposed in Yu and Jones [43]. The bandwidths for the kernel estimator for the conditional density in the bootstrap procedure were chosen by a rule of thumb using the function $b w . n r d$ in R. The level of the tests was set to 0.05. We observe in Table 4 that the level of our test is close to the nominal level. None of the two tests is always more powerful than the other. In the null model, both tests keep the level well. In scenario II, Zheng's test shows stronger power than the proposed test, whereas in scenario III(b), the proposed test has higher power.

Finally, as an illustrating example, we applied the proposed test to a historic data set of Ernst Engel. The data set was used in Koenker [30], among many other publications. The data set was first presented by Engel [12] to support his famous Engel's law. The data set has two variables, household income and food expenditure and it contains 235 observations. Figure 3 shows the scatter plot of this dataset and the scatter plot of the data after a log transform with base 10 . As one can see in Figure 3, there is one outlier. We removed this point from the data. Hence, the analysis below is based on 234 observations. We first analyzed the log transformed income versus 
Table 4. Rejection proportions based on 500 generated samples

\begin{tabular}{llllll}
\hline \multirow{5}{*}{} & \multicolumn{4}{l}{ Quartile functions } & \\
\cline { 3 - 6 } & & 1st quartile & Median & 3rd quartile & Sum quartiles \\
\hline Scenario I & Zheng & 0.006 & 0.000 & 0.000 & . \\
\multirow{2}{*}{ Scenario II } & MVY & 0.024 & 0.052 & 0.048 & 0.028 \\
& Zheng & 0.954 & 0.008 & 0.950 & . \\
Scenario III(a) & MVY & 0.696 & 0.002 & 0.650 & 0.884 \\
& Zheng & 0.974 & 0.994 & 0.984 & . \\
Scenario III(b) & MVY & 0.994 & 0.992 & 0.990 & 1.000 \\
& Zheng & 0.034 & 0.334 & 0.460 &. \\
& MVY & 0.048 & 0.404 & 0.992 & 0.954 \\
\hline
\end{tabular}
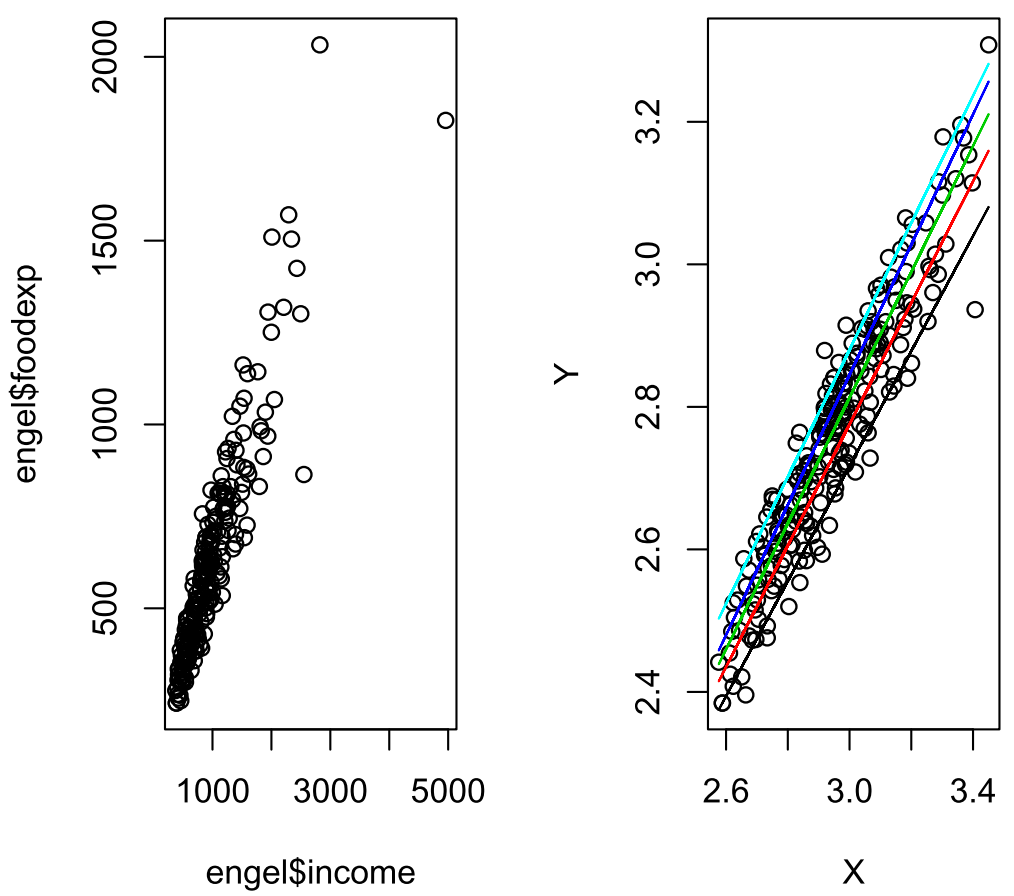

Figure 3. The left panel shows the scatter plot of the original Engel data. The right panel shows the scatter plot of log transformed data after removing one influential point. The lines in the right panel represent linear quantile fits of levels $0.1,0.3,0.5,0.7$, and 0.9 . 
Table 5. Estimated $p$-values for testing the linearity of conditional quantiles of Engel's data. The upper table shows the estimated $p$-values for testing the linearity of conditional quantiles of $\log _{10}$ (food expenditure) as a function of $\log _{10}$ (income) and the lower table shows the estimated $p$-values for testing the linearity of conditional quantiles of food expenditure as a function of $\log _{10}$ (income)

\begin{tabular}{|c|c|c|c|c|c|c|c|c|c|}
\hline \multirow[b]{2}{*}{ Bandwidth } & \multicolumn{9}{|c|}{ Quantile level } \\
\hline & 0.1 & 0.2 & 0.3 & 0.4 & 0.5 & 0.6 & 0.7 & 0.8 & 0.9 \\
\hline \multicolumn{10}{|c|}{$\log _{10}$ (income) vs $\log _{10}$ (food expenditure $)$} \\
\hline 0.050 & 0.289 & 0.246 & 0.629 & 0.999 & 0.996 & 1.000 & 0.999 & 1.000 & 1.000 \\
\hline 0.075 & 0.152 & 0.383 & 0.997 & 0.992 & 0.993 & 0.979 & 1.000 & 1.000 & 1.000 \\
\hline 0.100 & 0.105 & 0.745 & 0.997 & 0.964 & 0.971 & 0.970 & 0.999 & 1.000 & 0.993 \\
\hline 0.125 & 0.100 & 0.986 & 0.988 & 0.908 & 0.895 & 0.992 & 1.000 & 1.000 & 0.963 \\
\hline 0.150 & 0.149 & 0.996 & 0.976 & 0.894 & 0.843 & 0.997 & 0.999 & 1.000 & 0.935 \\
\hline \multicolumn{10}{|c|}{$\log _{10}$ (income) vs food expenditure } \\
\hline 0.050 & 0.305 & 0.675 & 0.413 & 0.135 & 0.031 & 0.008 & 0.012 & 0.004 & 0.000 \\
\hline 0.075 & 0.569 & 0.498 & 0.268 & 0.140 & 0.046 & 0.027 & 0.003 & 0.002 & 0.000 \\
\hline 0.100 & 0.640 & 0.324 & 0.181 & 0.047 & 0.015 & 0.004 & 0.003 & 0.001 & 0.000 \\
\hline 0.125 & 0.531 & 0.235 & 0.150 & 0.034 & 0.009 & 0.001 & 0.002 & 0.001 & 0.000 \\
\hline 0.150 & 0.664 & 0.197 & 0.088 & 0.012 & 0.003 & 0.002 & 0.002 & 0.003 & 0.000 \\
\hline
\end{tabular}

the log transformed food expenditure. We used five different bandwidths for calculating the test statistic. The bandwidth for the conditional density estimator used in the bootstrap resampling was chosen by a rule of thumb. To obtain the bootstrap distribution, we resampled the data set 1001 times. We test the linearity of quantiles for $\alpha=0.1,0.2,0.3,0.4,0.5,0.6,0.7,0.8$, and 0.9. As can be seen from Table 5, the test did not reject linearity of quantiles for any of these values at the significance level $5 \%$. This was the case for all five bandwidth choices. We also used the log transformed income with the original untransformed food expenditure as a further example. Figure 4 shows the scatter plot of this dataset together with 0.1, 0.3, 0.5, 0.7, 0.9 linear quantile fits. The figure shows that high level quantiles deviate from their linear fits. This is also seen by our test, since it rejects the linearity for high level quantiles. The estimated $p$-values for the conditional quantiles of level 0.5 or higher are smaller than 0.05 for every bandwidth we used.

\section{Proof of Theorem 1}

We need to show equations (11)-(13). Claim (13) follows from (11)-(12) because of $\Delta_{\alpha}^{h}(x)=$ $O\left(n^{-1 / 2} h^{-d / 4}\right)$. Furthermore, (14) is a direct consequence of (11), see the remark after the statement of the theorem. It remains to show (11)-(12).

It holds that $P\left(c m_{0} \leq N^{-}(x) \leq C m_{0}\right) \rightarrow 1$, where we use the shorthand notation $m_{0}=n h^{d}$. At this point and in the following proofs, we will make use of our convention of using the symbols, $c, C, \ldots$. 


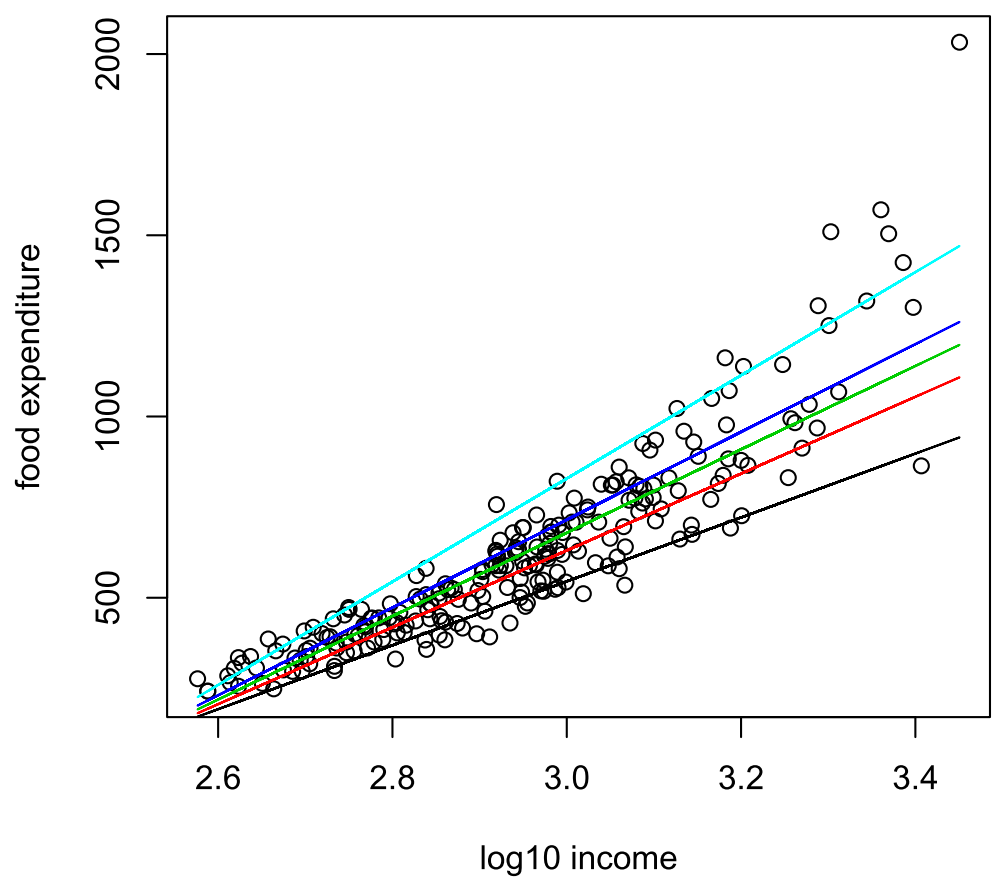

Figure 4. The figure shows the scatter plot of log transformed income versus food expenditure after removing one influential point. The lines represent linear quantile fits of level 0.1, 0.3, 0.5, 0.7, and 0.9.

First, note that

$$
\begin{aligned}
& \widehat{r}_{\alpha}^{\Delta,-}(x) \leq u m_{0}^{-1 / 2} \quad \text { if and only if } \\
& \sum_{j \in \mathcal{N}^{-}(x)} K\left(\frac{x-X_{j}}{h}\right)\left\{I\left(\varepsilon_{j, \alpha}^{\Delta} \leq \Delta_{\alpha}^{h}(x)+u m_{0}^{-1 / 2}\right)-\alpha\right\} \geq 0 .
\end{aligned}
$$

Let

$$
\begin{aligned}
g_{x, \alpha}(u)= & E_{x}^{j}\left[K\left(\frac{x-X_{j}}{h}\right)\left\{I\left(\varepsilon_{j, \alpha}^{\Delta} \leq \Delta_{\alpha}^{h}(x)+u m_{0}^{-1 / 2}\right)-\alpha\right\}\right] \\
= & E_{x}^{j}\left[K\left(\frac{x-X_{j}}{h}\right)\left\{I\left(\varepsilon_{j, \alpha} \leq \Delta_{\alpha}^{h}\left(x, X_{j}\right)+u m_{0}^{-1 / 2}\right)-\alpha\right\}\right] \\
= & u m_{0}^{-1 / 2} E_{x}^{j}\left[K\left(\frac{x-X_{j}}{h}\right) f_{\varepsilon_{\alpha} \mid X}\left(\Delta_{\alpha}^{h}\left(x, X_{j}\right) \mid X_{j}\right)\right] \\
& +\frac{1}{2} u^{2} m_{0}^{-1} E_{x}^{j}\left[K\left(\frac{x-X_{j}}{h}\right) f_{\varepsilon_{\alpha} \mid X}^{\prime}\left(\Delta_{\alpha}^{h}\left(x, X_{j}\right) \mid X_{j}\right)\right]+O\left(L_{n} m_{0}^{-3 / 2}\right)
\end{aligned}
$$


uniformly in $|u| \leq C^{*} L_{n}^{*}$, because of Assumption (B2). Then, with

$$
\eta_{j, \alpha, u, x}=K\left(\frac{x-X_{j}}{h}\right)\left\{I\left(\varepsilon_{j, \alpha}^{\Delta} \leq \Delta_{\alpha}^{h}(x)+u m_{0}^{-1 / 2}\right)-\alpha\right\}-g_{x, \alpha}(u),
$$

we have that

$$
\begin{aligned}
& P\left(\widehat{r}_{\alpha}^{\Delta,-}(x) \leq u m_{0}^{-1 / 2} \mid \mathcal{N}^{-}(x), N^{-}(x)=m\right) \\
& \quad=P\left(m^{-1 / 2} \sum_{j \in \mathcal{N}^{-}(x)} \eta_{j, \alpha, u, x} \geq-m^{1 / 2} g_{x, \alpha}(u) \mid \mathcal{N}^{-}(x), N^{-}(x)=m\right) .
\end{aligned}
$$

We now argue that an Edgeworth expansion holds for the conditional density of $T_{\eta}=$ $m^{-1 / 2} \sum_{j \in \mathcal{N}^{-}(x)} \eta_{j, \alpha, u, x}$, given $\mathcal{N}^{-}(x), N^{-}(x)=m$, that is of the form

$$
\sigma^{-1} \sum_{r=0}^{s-3} m^{-r / 2} P_{r}\left(-\phi:\left\{\chi_{\nu}\right\}\right)\left(\sigma^{-1}[\cdot-x]\right)+O\left(m^{-(s-2) / 2}\left[1+\left|\sigma^{-1}[\cdot-x]\right|^{s}\right]^{-1}\right),
$$

where the error term holds uniformly in $\alpha \in A,|u| \leq L_{n}^{*}$ and $x \in R_{X}$ for $C_{1}^{*} m_{0} \leq m \leq C_{2}^{*} m_{0}$ and constants $C_{1}^{*}<C_{2}^{*}$. Here, we use standard notation used for example, in Bhattacharya and Ranga Rao [3], page 53. In particular, $\sigma^{2}$ denotes the conditional variance of $\eta_{j, \alpha, u, x}$, given that $j \in \mathcal{N}^{-}(x)$, and $P_{r}\left(-\phi:\left\{\chi_{\nu}\right\}\right)$ denotes a product of a standard normal density $\phi$ with a polynomial that has coefficients depending only on the conditional cumulants $\chi_{\nu}$ of $\eta_{j, \alpha, u, x}$ of order $v \leq s-1$, given that $j \in \mathcal{N}^{-}(x)$. Note that $\sigma^{2}$ and $\chi_{\nu}$ depend on $u, \alpha, x$ and $n$ and that we do not indicate this in our notation. Furthermore, the cumulants and the variance converge to constants depending on $\alpha$, uniformly in $|u| \leq L_{n}^{*}$ and $x \in R_{X}$. Note that for $n \rightarrow \infty$ the conditional distribution of $\eta_{j, \alpha, u, x}$, given that $j \in \mathcal{N}^{-}(x)$, converges to the distribution of $K(U)(Z-\alpha)$ where $U$ and $Z$ are independent random variables, $U$ has a uniform distribution on $[-1,1]$ and $Z$ is $\{0,1\}$-valued with $P(Z=1)=\alpha$. This helps to understand that limit theorems hold uniformly. The function $P_{r}\left(-\phi:\left\{\chi_{\nu}\right\}\right)$ is defined as

$$
P_{r}\left(-\phi:\left\{\chi_{\nu}\right\}\right)(u)=\sum_{m=1}^{r} \frac{1}{m !}(-1)^{r} \sum_{j_{1}+\cdots+j_{m}=r} \frac{\chi_{j_{1}+2}}{\left(j_{1}+2\right) !} \cdot \cdots \cdot \frac{\chi_{j_{m}+2}}{\left(j_{m}+2\right) !} \phi^{(r+2 m)}(u),
$$

see Section 7 in Bhattacharya and Ranga Rao [3]. In our case expansion, (16) follows from Theorem 19.3 in Bhattacharya and Ranga Rao [3]. For this claim, we have to verify that their conditions (19.27), (19.29) and (19.30) hold. Our setting is slightly different from theirs, since we consider triangular arrays of independent identically distributed random variables instead of a sequence of independent random variables as is the case in Theorem 19.3 in Bhattacharya and Ranga Rao [3]. But the same proof applies because in our setting we can verify the following uniform versions of (19.27), (19.29) and (19.30):

$$
\sup _{\alpha \in A,|u| \leq L_{n}^{*}, x \in R_{X}, n \geq n_{0}} E_{x}^{j}\left[\left|\eta_{j, \alpha, u, x}\right|^{s}\right]<\infty,
$$




$$
\begin{aligned}
\sup _{\alpha \in A,|u| \leq L_{n}^{*}, x \in R_{X}, n \geq n_{0}} \int g_{\alpha, u, x}^{q}(t) d t<\infty & \text { for some } q>0, \\
\sup _{\alpha \in A,|u| \leq L_{n}^{*}, x \in R_{X}, n \geq n_{0}}\left\{g_{\alpha, u, x}(t):|t| \geq b\right\}<1 & \text { for all } b>0
\end{aligned}
$$

with $g_{\alpha, u, x}(t)=\left|E_{x}^{j}\left[\exp \left(i t \sigma^{-1} \eta_{j, \alpha, u, x}\right)\right]\right|$ for some $n_{0}>0$. Note that $g_{\alpha, u, x}, \sigma$ and $\eta_{j, \alpha, u, x}$ depend on $n$.

Claim (17) follows by a direct argument using brute force bounds. For the proof of (18), we consider the conditional density of $U_{p}=\sum_{j=1}^{p}\left(k\left(\frac{x_{1}-X_{1, j}}{h}\right), \ldots, k\left(\frac{x_{d}-X_{d, j}}{h}\right)\right)^{\top}\left\{I\left(\varepsilon_{j, \alpha}^{\Delta} \leq\right.\right.$ $\left.\left.\Delta_{\alpha}^{h}(x)+u m_{0}^{-1 / 2}\right)-\alpha\right\}$ given the value of $\varepsilon_{j, \alpha}^{\Delta}$ and given that $X_{j} \in \mathcal{N}^{-}(x)$ for $j=1, \ldots, p$. For $p=1$ this density evaluated at $\left(u_{1}, \ldots, u_{d}\right)$ can be bounded by a constant times $\left(u_{1} \cdot \ldots \cdot u_{d}\right)^{-\kappa}\left(\left(k(0)-u_{1}\right) \cdot \ldots \cdot\left(k(0)-u_{d}\right)\right)^{-\kappa}$ by Assumptions (B1) and (B3). This bound holds uniformly over $\alpha, u, x$ and the value of $\varepsilon_{1, \alpha}^{\Delta}$.

We now show that for every $\kappa^{*}>0$, there exists $p^{*}>0$ such that the density of $U_{p^{*}}$ can be bounded by a constant times $\left(u_{1} \cdot \ldots \cdot u_{d}\right)^{\kappa^{*}}$. For simplification of notation, we assume for the proof that $d=1$. For the proof of the claim, we show first that for $p \geq(1-\kappa)^{-1}$ we get that the conditional density of $U_{p}$ is uniformly bounded. This follows by an evaluation of convolution integrals where one uses that $\int_{0}^{v} u^{-\kappa_{1}}(v-u)^{-\kappa_{2}} d u=v^{-\left(\kappa_{1}+\kappa_{2}-1\right)} \int_{0}^{1} w^{-\kappa_{1}} \times$ $(1-w)^{-\kappa_{2}} d w \leq C v^{-\left(\kappa_{1}+\kappa_{2}-1\right)}$. Applied to $U_{2}$ this gives that the density of $U_{2}$ is bounded by $C u_{1}^{-(2 \kappa-1)}+C\left|k(0)-u_{1}\right|^{-(2 \kappa-1)}+C\left(2 k(0)-u_{1}\right)^{-(2 \kappa-1)}$. Note that the density of $U_{1}$ is bounded by $C u_{1}^{-\kappa}\left(k(0)-u_{1}\right)^{-\kappa} \leq C u_{1}^{-\kappa}+C\left(k(0)-u_{1}\right)^{-\kappa}$. For the density of $U_{3}$ we get the bound $C u_{1}^{-(3 \kappa-2)}+C\left|k(0)-u_{1}\right|^{-(3 \kappa-2)}+C\left|2 k(0)-u_{1}\right|^{-(3 \kappa-2)}+C\left(3 k(0)-u_{1}\right)^{-(3 \kappa-2)}$. Finally, for $p \geq(1-\kappa)^{-1}$ it holds that $p \kappa-(p-1) \leq 0$ and we have that for $p \geq(1-\kappa)^{-1}$, the density of $U_{p}$ is uniformly bounded. We now use that the $k$-fold convolution of a bounded density with bounded support $[0, z]$ for some $z>0$ is bounded by $C\left|u_{1}\right|^{k-1}$. This gives that the density of $U_{p^{*}}$ can be bounded by a constant times $u_{1}^{\kappa^{*}}$ if $p^{*} \leq l_{0}\left(\kappa^{*}+1\right)$ with $l_{0} \geq(1-\kappa)^{-1}$, $l \in \mathbb{N}$. This result can be easily extended to $d>1$.

From this result, now we want to conclude that the conditional density of $\sum_{j=1}^{p} \eta_{j, \alpha, u, x}=$ $\sum_{j=1}^{p} K\left(\frac{x-X_{j}}{h}\right)\left\{I\left(\varepsilon_{j, \alpha}^{\Delta} \leq \Delta_{\alpha}^{h}(x)+u m_{0}^{-1 / 2}\right)-\alpha\right\}=\sum_{j=1}^{p} k\left(\frac{x_{1}-X_{1, j}}{h}\right) \cdot \ldots \cdot k\left(\frac{x_{d}-X_{d, j}}{h}\right)\left\{I\left(\varepsilon_{j, \alpha}^{\Delta} \leq\right.\right.$ $\left.\left.\Delta_{\alpha}^{h}(x)+u m_{0}^{-1 / 2}\right)-\alpha\right\}$ is uniformly bounded, given the value of $\varepsilon_{j, \alpha}^{\Delta}$ and given that $X_{j} \in$ $\mathcal{N}^{-}(x)$ for $j=1, \ldots, p$. This follows immediately from the following result. Suppose that $Z=\left(Z_{1}, \ldots, Z_{d}\right)$ has support $[0,1]^{d}$ and a density $f$ that is bounded by $f(z) \leq D z_{1} \cdot \ldots \cdot z_{d}$ then $Z_{1} \cdot \ldots \cdot Z_{d}$ has a density $g$ that is bounded by $D$. For a proof of this result, note that

$$
\begin{aligned}
g(u) & =\partial_{u} \int_{z_{1} \cdot \ldots \cdot z_{d} \leq u} f(z) d z_{1} \cdots d z_{d} \\
& =\partial_{u} \int_{v \leq u} f\left(\frac{v}{z_{2} \cdot \ldots \cdot z_{d}}, z_{2}, \ldots, z_{d}\right) \frac{1}{z_{2} \cdot \ldots \cdot z_{d}} d v d z_{2} \cdots d z_{d} \\
& =\int f\left(\frac{u}{z_{2} \cdot \ldots \cdot z_{d}}, z_{2}, \ldots, z_{d}\right) \frac{1}{z_{2} \cdot \ldots \cdot z_{d}} d z_{2} \cdots d z_{d}
\end{aligned}
$$




$$
\begin{aligned}
& \leq \int f\left(\frac{u}{z_{2} \cdot \ldots \cdot z_{d}}, z_{2}, \ldots, z_{d}\right) \frac{1}{u} d z_{2} \cdots d z_{d} \\
& \leq \int D \frac{u}{z_{2} \cdot \ldots \cdot z_{d}} \cdot z_{2} \cdot \ldots \cdot z_{d} \cdot \frac{1}{u} d z_{2} \cdots d z_{d} \\
& =D .
\end{aligned}
$$

We now apply the result that for $p$ chosen large enough, the conditional density of $\sum_{j=1}^{p} \eta_{j, \alpha, u, x}$ is bounded, given that $X_{j} \in \mathcal{N}^{-}(x)$ for $j=1, \ldots, p$, uniformly over $\alpha, u$ and $x$. This implies that the square of this conditional density is integrable and by the Fourier Inversion theorem (see Theorem 4.1(vi) in Bhattacharya and Ranga Rao [3]) the same holds for the squared modulus of its Fourier transform. Thus, the modulus of the Fourier transform of the conditional density of $\sum_{j=1}^{2 p} \eta_{j, \alpha, u, x}$, given that $X_{j} \in \mathcal{N}^{-}(x)$ for $j=1, \ldots, 2 p$, is integrable. This shows (18) for $q=2 p$.

For the proof of (19) one applies the Riemann-Lebesgue lemma (see Theorem 4.1 in Bhattacharya and Ranga Rao [3]). Consider for simplicity the case where $d=1$. For $E_{x}^{j}\left[\exp \left(i t \eta_{j, \alpha, u, x}\right)\right]$, one gets that

$$
\begin{aligned}
\exp [ & \left.\operatorname{itg}_{x, \alpha}(u)\right] E_{x}^{j}\left[\exp \left(\text { it }_{j, \alpha, u, x}\right)\right] \\
= & \int_{x-h}^{x+h} \int_{e \in \mathbb{R}} \exp \left[i t K\left(\frac{x-z}{h}\right)\left\{\mathrm{I}\left(e \leq \Delta_{\alpha}^{h}(x, z)+u m_{0}^{-1 / 2}\right)-\alpha\right\}\right] \\
& \times f_{\varepsilon_{\alpha} \mid X}(e \mid z) f_{X}(z) d e d z / \int_{x-h}^{x+h} f_{X}(z) d z \\
= & \int_{-1}^{1} \int_{e \in \mathbb{R}} \exp \left[i t K(v)\left\{\mathrm{I}\left(e \leq \Delta_{\alpha}^{h}(x, x+h v)+u m_{0}^{-1 / 2}\right)-\alpha\right\}\right] \\
& \times f_{\varepsilon_{\alpha} \mid X}(e \mid x+h v) f_{X}(x+h v) d e d v / \int_{-1}^{1} f_{X}(x+h v) d v .
\end{aligned}
$$

The right-hand side of this equation converges to

$$
\alpha \int_{-1}^{1} \exp [i t(1-\alpha) K(v)] d v+(1-\alpha) \int_{-1}^{1} \exp [-i t \alpha K(v)] d v .
$$

This convergence holds uniformly in $t \in \mathbb{R}, \alpha \in A,|u| \leq L_{n}^{*} m_{0}^{-1 / 2}$ and $x \in R_{X}$. By using these facts, we get (19) from the Riemann-Lebesgue lemma.

By applying Theorem 19.3 in Bhattacharya and Ranga Rao [3] with $s \geq 4$, we get that

$$
\begin{aligned}
& P\left(\widehat{r}_{\alpha}^{\Delta,-}(x) \leq u m_{0}^{-1 / 2} \mid \mathcal{N}^{-}(x), N^{-}(x)=m\right) \\
& \quad=1-\Phi\left(\mu_{\alpha}(u)\right)+m^{-1 / 2} \rho_{\alpha}(u)\left(1-\mu_{\alpha}(u)^{2}\right) \phi\left(\mu_{\alpha}(u)\right)+O\left(m_{0}^{-1}\left(1+\mu_{\alpha}(u)^{2}\right)^{-s}\right)
\end{aligned}
$$

uniformly in $u, \alpha$ and $x$ for $C_{1}^{*} m_{0} \leq m \leq C_{2}^{*} m_{0}$ and constants $C_{1}^{*}<C_{2}^{*}$. Here we have used the fact that terms for $r=2, \ldots, s-3$ in the expansion (16) can be bounded by $O\left(m_{0}^{-1}(1+\right.$ 
$\left.\mu_{\alpha}(u)^{2}\right)^{-s}$ ). We used the following notation

$$
\mu_{\alpha}(u)=-\frac{m^{1 / 2} g_{x, \alpha}(u)}{\sigma_{\alpha}(u)} \quad \text { and } \quad \rho_{\alpha}(u)=\frac{E_{x}^{j}\left(\eta_{j, \alpha, u, x}^{3}\right)}{6 \sigma_{\alpha}^{3}(u)}
$$

with $\sigma_{\alpha}^{2}(u)=E_{x}^{j}\left(\eta_{j, \alpha, u, x}^{2}\right)$. It is easy to show that, uniformly in $|u| \leq C^{*} L_{n}^{*}$,

$$
\begin{aligned}
\sigma_{\alpha}^{2}(u)= & E_{x}^{j}\left[K^{2}\left(\frac{x-X_{i}}{h}\right)\left(I\left(\varepsilon_{j, \alpha} \leq \Delta_{\alpha}^{h}\left(x, X_{j}\right)+u m_{0}^{-1 / 2}\right)-\alpha\right)^{2}\right] \\
& +O\left(L_{n} m_{0}^{-1}\right) \\
= & A_{1}(\alpha)+u m_{0}^{-1 / 2} A_{2}(\alpha)+O\left(L_{n} m_{0}^{-1}\right), \\
E_{x}^{j}\left(\eta_{j, \alpha, u, x}^{3}\right)= & E_{x}^{j}\left[K^{3}\left(\frac{x-X_{i}}{h}\right)\left(I\left(\varepsilon_{j, \alpha} \leq \Delta_{\alpha}^{h}\left(x, X_{j}\right)+u m_{0}^{-1 / 2}\right)-\alpha\right)^{3}\right] \\
& +O\left(L_{n} m_{0}^{-1 / 2}\right) \\
= & A_{3}(\alpha)+O\left(L_{n} m_{0}^{-1 / 2}\right),
\end{aligned}
$$

and that

$$
\begin{aligned}
\mu_{\alpha}(u)= & -u m^{1 / 2} m_{0}^{-1 / 2} A_{1}(\alpha)^{-1 / 2} A_{5}(\alpha)-\frac{1}{2} u^{2} m^{1 / 2} m_{0}^{-1} A_{1}(\alpha)^{-1 / 2} A_{6}(\alpha) \\
& +\frac{1}{2} u^{2} m^{1 / 2} m_{0}^{-1} A_{1}^{-3 / 2}(\alpha) A_{2}(\alpha) A_{5}(\alpha)+O\left(L_{n} m_{0}^{-1}\right)
\end{aligned}
$$

with

$$
\begin{aligned}
& A_{1}(\alpha)=E_{x}^{j}\left[K^{2}\left(\frac{x-X_{i}}{h}\right)\left((1-2 \alpha) P\left(\varepsilon_{j, \alpha} \leq \Delta_{\alpha}^{h}\left(x, X_{j}\right)\right)+\alpha^{2}\right)\right], \\
& A_{2}(\alpha)=E_{x}^{j}\left[K^{2}\left(\frac{x-X_{i}}{h}\right)(1-2 \alpha) f_{\varepsilon_{\alpha} \mid X}\left(\Delta_{\alpha}^{h}\left(x, X_{j}\right) \mid X_{j}\right)\right], \\
& A_{3}(\alpha)=E_{x}^{j}\left[K^{3}\left(\frac{x-X_{i}}{h}\right)\left(\left(1-3 \alpha+3 \alpha^{2}\right) P\left(\varepsilon_{j, \alpha} \leq \Delta_{\alpha}^{h}\left(x, X_{j}\right)\right)-\alpha^{3}\right)\right], \\
& A_{4}(\alpha)=E_{x}^{j}\left[K^{2}\left(\frac{x-X_{i}}{h}\right)\left((1-2 \alpha) P\left(\varepsilon_{j, \alpha} \leq \Delta_{\alpha}^{h}\left(x, X_{j}\right)\right)+\alpha^{2}\right)\right], \\
& A_{5}(\alpha)=E_{x}^{j}\left[K\left(\frac{x-X_{j}}{h}\right) f_{\varepsilon_{\alpha} \mid X}\left(\Delta_{\alpha}^{h}\left(x, X_{j}\right) \mid X_{j}\right)\right], \\
& A_{6}(\alpha)=E_{x}^{j}\left[K\left(\frac{x-X_{j}}{h}\right) f_{\varepsilon_{\alpha} \mid X}^{\prime}\left(\Delta_{\alpha}^{h}\left(x, X_{j}\right) \mid X_{j}\right)\right] .
\end{aligned}
$$


Note that $\mu_{\alpha}(-u)^{2}=\mu_{\alpha}(u)^{2}+O\left(L_{n} m_{0}^{-1 / 2}\right)$. Thus, we get that uniformly in $|u| \leq C^{*} L_{n}^{*}$,

$$
\begin{aligned}
& m^{-1 / 2} \rho_{\alpha}(u)\left(1-\mu_{\alpha}(u)^{2}\right) \phi\left(\mu_{\alpha}(u)\right)-m^{-1 / 2} \rho_{\alpha}(-u)\left(1-\mu_{\alpha}(-u)^{2}\right) \phi\left(\mu_{\alpha}(-u)\right) \\
& \quad=O\left(L_{n} m_{0}^{-1}\right) \\
& m^{-1 / 2} \rho_{\alpha}(u)\left(1-\mu_{\alpha}(u)^{2}\right) \phi\left(\mu_{\alpha}(u)\right)=O\left(L_{n} m_{0}^{-1 / 2}\right)
\end{aligned}
$$

Note also that with $u_{m}=u m^{1 / 2} m_{0}^{-1 / 2}$, uniformly in $|u| \leq C^{*} L_{n}^{*}$,

$$
\begin{aligned}
1-\Phi\left(\mu_{\alpha}(u)\right)= & 1-\Phi\left(-u_{m} A_{1}^{-1 / 2}(\alpha) A_{5}(\alpha)\right) \\
& +\phi\left(-u_{m} A_{1}^{-1 / 2}(\alpha) A_{5}(\alpha)\right) \frac{u_{m}^{2}}{2 m^{1 / 2}}\left(A_{1}^{-1 / 2}(\alpha) A_{6}(\alpha)-A_{1}^{-3 / 2}(\alpha) A_{2}(\alpha) A_{5}(\alpha)\right) \\
& +O\left(L_{n} m_{0}^{-1}\right) .
\end{aligned}
$$

Hence, uniformly in $|u| \leq C^{*} L_{n}^{*}$,

$$
1-\Phi\left(\mu_{\alpha}(u)\right)+\Phi\left(-\mu_{\alpha}(-u)\right)=2\left[1-\Phi\left(-u_{m} A_{1}^{-1 / 2}(\alpha) A_{5}(\alpha)\right)\right]+O\left(L_{n} m_{0}^{-1}\right) .
$$

From (21), (23) and the above calculations it now follows for $l \geq 1$ that with $D_{m}(\alpha)=$ $m^{1 / 2} m_{0}^{-1 / 2} A_{1}^{-1 / 2}(\alpha) A_{5}(\alpha)$ and $\widehat{r}_{\alpha}^{\Delta,-}(x)=\widehat{r}_{\alpha}^{\Delta}(x)-\Delta_{\alpha}^{h}(x)$

$$
\begin{aligned}
E\left\{m_{0}^{l} \widehat{r}_{\alpha}^{\Delta,-}(x)^{2 l} I\left(\left|\widehat{r}_{\alpha}^{\Delta,-}(x)\right| \leq L_{n}^{*} m_{0}^{-1 / 2}\right) \mid N^{-}(x)=m\right\} \\
=2 l \int_{0}^{L_{n}^{*}} v^{2 l-1} P\left(\widehat{r}_{\alpha}^{\Delta,-}(x)>v m_{0}^{-1 / 2} \mid N^{-}(x)=m\right) d v \\
\quad-2 l \int_{-L_{n}^{*}}^{0} v^{2 l-1} P\left(\widehat{r}_{\alpha}^{\Delta,-}(x) \leq v m_{0}^{-1 / 2} \mid N^{-}(x)=m\right) d v \\
=2 l \int_{0}^{L_{n}^{*}} v^{2 l-1}\left[P\left(\widehat{r}_{\alpha}^{\Delta,-}(x)>v m_{0}^{-1 / 2} \mid N^{-}(x)=m\right)\right. \\
\left.\quad+P\left(\widehat{r}_{\alpha}^{\Delta,-}(x) \leq-v m_{0}^{-1 / 2} \mid N^{-}(x)=m\right)\right] d v \\
=2 l \int_{0}^{L_{n}^{*}} v^{2 l-1}\left[\Phi\left(\mu_{\alpha}(v)\right)-m^{-1 / 2} \rho_{\alpha}(v)\left(1-\mu_{\alpha}(v)^{2}\right) \phi\left(\mu_{\alpha}(v)\right)\right. \\
\left.\quad+1-\Phi\left(\mu_{\alpha}(-v)\right)+m^{-1 / 2} \rho_{\alpha}(-v)\left(1-\mu_{\alpha}(-v)^{2}\right) \phi\left(\mu_{\alpha}(-v)\right)\right] d v \\
\quad+O\left(L_{n} m_{0}^{-1}\right) \\
=4 l \int_{0}^{L_{n}^{*}} v^{2 l-1} \Phi\left(-v D_{m}(\alpha)\right) d v+O\left(L_{n} m_{0}^{-1}\right) \\
=4 l D_{m}(\alpha)^{-2 l} \int_{0}^{L_{n}^{*} D_{m}(\alpha)} w^{2 l-1} \Phi(-w) d w+O\left(L_{n} m_{0}^{-1}\right) \\
\quad
\end{aligned}
$$




$$
\begin{aligned}
= & 2\left[\left(L_{n}^{*}\right)^{2 l} \Phi\left(-L_{n}^{*} D_{m}(\alpha)\right)+D_{m}(\alpha)^{-2 l} \int_{0}^{L_{n}^{*} D_{m}(\alpha)} v^{2 l} \phi(v) d v\right] \\
& +O\left(L_{n} m_{0}^{-1}\right)
\end{aligned}
$$

uniformly in $C_{1}^{*} m_{0} \leq m \leq C_{2}^{*} m_{0}$ with constants $C_{1}^{*}<C_{2}^{*}$. If $L_{n}^{*}=(\log n)^{\gamma}$ is chosen with $\gamma>0$ large enough, we get that the right-hand side of the last equation is equal to $D_{m}(\alpha)^{-2 l} \int_{-\infty}^{\infty} v^{2 l} \phi(v) d v+O\left(L_{n} m_{0}^{-1}\right)$. This follows since it can be easily shown that

$$
\begin{array}{r}
2 \int_{0}^{L_{n}^{*} D_{m}(\alpha)} z^{2 l} \phi(z) d z-\int_{-\infty}^{\infty} z^{2 l} \phi(z) d z=o\left(L_{n} n^{-C^{*}}\right)=o\left(m^{-C^{*}}\right), \\
\left(L_{n}^{*}\right)^{2 l} \Phi\left(-L_{n}^{*} D_{m}(\alpha)\right)=o\left(m^{-C^{*}}\right)
\end{array}
$$

with $\gamma$ chosen depending on $C^{*}$. Thus, we get for $l \in \mathbb{N}$ that

$$
\begin{gathered}
E\left\{\widehat{r}_{\alpha}^{\Delta,-}(x)^{2 l} I\left(\left|\widehat{r}_{\alpha}^{\Delta,-}(x)\right| \leq L_{n}^{*} m_{0}^{-1 / 2}\right) \mid N^{-}(x)=m\right\} \\
\quad=m^{-l} \frac{A_{1}^{l}(\alpha)}{A_{5}^{2 l}(\alpha)} \int_{-\infty}^{\infty} z^{2 l} \phi(z) d z+O\left(L_{n}\left(n h^{d}\right)^{-l-1}\right) .
\end{gathered}
$$

With similar arguments one can show that

$$
\begin{aligned}
E & \left\{\widehat{r}_{\alpha}^{\Delta,-}(x)^{2 l-1} I\left(\left|\widehat{r}_{\alpha}^{\Delta,-}(x)\right| \leq L_{n}^{*} m_{0}^{-1 / 2}\right) \mid N^{-}(x)=m\right\} \\
& =m^{-(2 l-1) / 2} \frac{A_{1}^{(2 l-1) / 2}(\alpha)}{A_{5}^{2 l-1}(\alpha)} \int_{-\infty}^{\infty} z^{2 l-1} \phi(z) d z+O\left(L_{n}\left(n h^{d}\right)^{-l}\right) \\
& =O\left(L_{n}\left(n h^{d}\right)^{-l}\right) .
\end{aligned}
$$

In this case, one applies (22) instead of (21).

For the proof of (11) and (12) it remains to show that uniformly in $C_{1}^{*} m_{0} \leq m \leq C_{2}^{*} m_{0}$, with constants $C_{1}^{*}<C_{2}^{*}$, and for $l \in \mathbb{N}$

$$
\begin{aligned}
E\left\{\widetilde{r}_{\alpha}^{\Delta,-}(x)^{2 l} \mid N^{-}(x)\right. & =m\}=m^{-\kappa / 2} \frac{A_{1}^{\kappa / 2}(\alpha)}{A_{5}^{\kappa}(\alpha)} \int_{-\infty}^{\infty} z^{2 l} \phi(z) d z+O\left(L_{n}\left(n h^{d}\right)^{-l-1}\right), \\
E\left\{\widetilde{r}_{\alpha}^{\Delta,-}(x)^{2 l-1} \mid N^{-}(x)=m\right\} & =O\left(L_{n}\left(n h^{d}\right)^{-l}\right) .
\end{aligned}
$$

It remains to show (26)-(27). For the proof of (26) note that for independent random variables $Z_{1}, \ldots, Z_{m}$ with mean zero, variance 1 and bounded $2 l$ th absolute moment it holds that

$$
E\left\{\left(m^{-1 / 2} \sum_{i=1}^{m} Z_{i}\right)^{2 l}\right\}=\int_{-\infty}^{\infty} z^{2 l} \phi(z) d z+O\left(m^{-1}\right)
$$


because for $Z_{1}^{*}, \ldots, Z_{m}^{*} \stackrel{\text { i.i.d. }}{\sim} N(0,1)$ one has

$$
\begin{aligned}
E\left\{\left(m^{-1 / 2} \sum_{i=1}^{m} Z_{i}\right)^{2 l}\right\} & =m^{-l} \sum^{*} E\left(Z_{i_{1}} \cdots Z_{i_{2 l}}\right)+O\left(m^{-1}\right) \\
& =m^{-l} \sum^{*} E\left(Z_{i_{1}}^{*} \cdots Z_{i_{2 l}}^{*}\right)+O\left(m^{-1}\right) \\
& =E\left\{\left(m^{-1 / 2} \sum_{i=1}^{m} Z_{i}^{*}\right)^{2 l}\right\}+O\left(m^{-1}\right) \\
& =E\left(\left(Z_{1}^{*}\right)^{2 l}\right)+O\left(m^{-1}\right) \\
& =\int_{-\infty}^{\infty} z^{2 l} \phi(z) d z+O\left(m^{-1}\right)
\end{aligned}
$$

where the sum $\sum^{*}$ runs over all indices $i_{1}, \ldots, i_{2 l}$ that are such that each value of an index appears exactly two times. For the proof of (27), one applies that for independent random variables $Z_{1}, \ldots, Z_{m}$ with mean zero, variance 1 and bounded $2 l+1$ th absolute moment it holds that

$$
\begin{aligned}
E\left\{\left(m^{-1 / 2} \sum_{i=1}^{m} Z_{i}\right)^{2 l-1}\right\} & =m^{-l+1} m^{-1 / 2} E\left(\sum_{i=1}^{m} Z_{i}\right)^{2 l-1} \\
& =m^{-l+1} m^{-1 / 2} \sum^{* *} E\left(Z_{i_{1}} \cdots Z_{i_{2 l-1}}\right)+O\left(m^{-3 / 2}\right)=O\left(m^{-1 / 2}\right),
\end{aligned}
$$

where the sum $\sum^{* *}$ runs over all indices that are such that one value of an index appears three times and for all other $2 l-4$ indices each value appears exactly two times. This concludes the proof of the theorem.

\section{Proof of Theorem 2}

For the proof of Theorem 2, we will use the following corollary of Theorem 1. For the statement of the corollary, we have to define another construction of local neighborhoods. For their definition, suppose first that $X$ is one-dimensional. Then the support $R_{X}$ is a compact interval. For arbitrary $j$ and for $k \in\{1,2,3\}$, we can then define

$$
I_{j k}=[(3 j+k-1) h,(3 j+k) h] \quad \text { and } \quad I_{j k}^{*}=[(3 j+k-2) h,(3 j+k+1) h] .
$$

The set of indices of the $X_{i}(i=1, \ldots, n)$ that fall inside the interval $I_{j k}^{*}$ is denoted by $\mathcal{N}_{j k}$. We write $N_{j k}$ for the number of elements of $\mathcal{N}_{j k}$. An arbitrary $x \in R_{X}$ belongs to a unique $I_{j k}$ and we define $\mathcal{N}(x)=\mathcal{N}_{j k}$ and $N(x)=N_{j k}$. Thus, $\mathcal{N}(x)$ is an interval of length $3 h$, such that $x$ lies in the middle subinterval of $\mathcal{N}(x)$ of length $h$. If the dimension of $X$ is larger than one, this partition of the support into small intervals can be generalized in an obvious way. 
Corollary 1. Assume (B1)-(B3). Then, for natural numbers $l \geq 1$,

$$
\begin{aligned}
E\left\{\bar{r}_{\alpha}^{\Delta,-}(x)^{2 l}-\widetilde{r}_{\alpha}^{\Delta,-}(x)^{2 l} \mid N(x)=m\right\} & =O\left(L_{n}\left(n h^{d}\right)^{-l-1}\right), \\
E\left\{\bar{r}_{\alpha}^{\Delta,-}(x)^{2 l-1}-\widetilde{r}_{\alpha}^{\Delta,-}(x)^{2 l-1} \mid N(x)=m\right\} & =O\left(L_{n}\left(n h^{d}\right)^{-l}\right)
\end{aligned}
$$

uniformly in $x \in R_{X}, \alpha \in A$ and $C_{1}^{*} n h^{d} \leq m \leq C_{2}^{*} n h^{d}$, where $N(x)$ is the random number of $X_{i}$ 's that lie in $\mathcal{N}(x)$, and where $\bar{r}_{\alpha}^{\Delta,-}(x)=\bar{r}_{\alpha}^{\Delta}(x)-\Delta_{\alpha}^{h}(x)$. For the second moments of the uncentered estimators $\bar{r}_{\alpha}^{\Delta}$ and $\widetilde{r}_{\alpha}^{\Delta}$, we have that

$$
E\left\{\bar{r}_{\alpha}^{\Delta}(x)^{2}-\widetilde{r}_{\alpha}^{\Delta}(x)^{2} \mid N(x)=m\right\}=O\left(L_{n} n^{-3 / 2} h^{-5 d / 4}\right) .
$$

Under the additional assumption that $\Delta_{\alpha} \equiv 0$, we get that

$$
E\left\{\bar{r}_{\alpha}^{\Delta}(x)^{2}-\widetilde{r}_{\alpha}^{\Delta}(x)^{2} \mid N(x)=m\right\}=O\left(L_{n}\left(n h^{d}\right)^{-2}\right)
$$

Proof. For $m^{+} \geq m$, we have by a simple argument with $\kappa=2 l$ or $\kappa=2 l+1$ that $E\left\{\bar{r}_{\alpha}^{\Delta,-}(x)^{\kappa}-\widetilde{r}_{\alpha}^{\bar{\Delta},-}(x)^{\kappa} \mid N(x)=m^{+}, N^{-}(x)=m\right\}=E\left\{\bar{r}_{\alpha}^{\Delta,-}(x)^{\kappa}-\widetilde{r}_{\alpha}^{\Delta,-}(x)^{\kappa} \mid N^{-}(x)=m\right\}$. Note that $N^{-}(x) \leq N(x)$ because of $\mathcal{N}^{-}(x) \subset \mathcal{N}(x)$. Using (11) and

$$
P\left(N^{-}(x) \leq \frac{m^{+}}{4} \mid N(x)=m^{+}\right) \leq C \exp \left(-c n h^{d}\right)
$$

uniformly in $m^{+} \geq \frac{1}{2} 3^{d} f_{X}(x) n h^{d}$ we conclude that

$$
E\left\{\bar{r}_{\alpha}^{\Delta,-}(x)^{\kappa}-\widetilde{r}_{\alpha}^{\Delta,-}(x)^{\kappa} \mid N(x)=m^{+}\right\}=O\left(L_{n}\left(n h^{d}\right)^{-l-1}\right)
$$

uniformly in $x \in R_{X}, \alpha \in A$ and $\frac{1}{2} 3^{d} f_{X}(x) n h^{d} \leq m^{+} \leq 23^{d} f_{X}(x) n h^{d}$.

Since

$$
P\left(\frac{1}{2} 3^{d} f_{X}(x) n h^{d} \leq N(x) \leq 23^{d} f_{X}(x) n h^{d} \text { for all } x \in R_{X}\right) \rightarrow 1,
$$

we get the statement of the corollary.

We now come to the proof of Theorem 2 .

We only prove the statement for $\widehat{T}_{A}$. The asymptotic result for $\widehat{T}_{\alpha}$ follows similarly. We need to introduce a few more notations. With $\delta_{\theta, \alpha}(x)=-\left(\theta(\alpha)-\theta_{0}(\alpha)\right)^{\top} \gamma_{\alpha}(x)+n^{-1 / 2} h^{-d / 4} \Delta_{\alpha}(x)$ and $\varepsilon_{i, \alpha}^{\Delta}=\varepsilon_{i, \alpha}+n^{-1 / 2} h^{-d / 4} \Delta_{\alpha}\left(X_{i}\right)$, we define $\widetilde{r}_{\alpha}^{\Delta}$ as in (9) and we put

$$
\widehat{r}_{\alpha, \theta}^{\Delta}(x)=\arg \min _{r} \sum_{i=1}^{n} K\left(\frac{x-X_{i}}{h}\right) \tau_{\alpha}\left(\varepsilon_{i . \alpha}+\delta_{\theta, \alpha}\left(X_{i}\right)-r\right) .
$$


Note that $\widehat{r}_{\alpha}^{\Delta}(x)=\widehat{r}_{\alpha, \theta_{0}}^{\Delta}(x)$, and that $\widehat{r}_{\alpha}(x)=\widehat{r}_{\alpha, \widehat{\theta}}^{\Delta}(x)+O_{P}\left(n^{-1 / 2-c}\right)$ by Assumption (B4). We also define $\bar{r}_{\alpha}^{\Delta}$ as in (8). Let also

$$
W_{n i}(x, h)=K_{h}\left(x-X_{i}\right) /\left[\sum_{j} K_{h}\left(x-X_{j}\right)\right]
$$

with $K_{h}(\cdot)=K(\cdot / h) / h^{d}$.

The proof of Theorem 2 will make use of the following lemmas.

Lemma 1. Suppose that the assumptions of Theorem 2 are satisfied. Then,

$$
\begin{aligned}
& \sup _{\alpha \in A} \sup _{x \in R_{X}}\left|\widehat{r}_{\alpha}(x)\right|=O_{P}\left(\left(n h^{d}\right)^{-1 / 2} L_{n}\right), \\
& \sup _{\alpha \in A} \sup _{x \in R_{X}}\left|\widehat{r}_{\alpha}^{\Delta}(x)\right|=O_{P}\left(\left(n h^{d}\right)^{-1 / 2} L_{n}\right) .
\end{aligned}
$$

Proof. As is known for the case where there is no parametric part and where $\Delta_{\alpha} \equiv 0$, one has that

$$
\sup _{\alpha \in A} \sup _{x \in R_{X}}\left|\widehat{r}_{\alpha, \theta_{0}}^{\Delta}(x)-\widetilde{r}_{\alpha}(x)\right|=O_{P}\left(\left(n h^{d}\right)^{-3 / 4} L_{n}\right)
$$

with $\widetilde{r}_{\alpha}$ defined as in (5). For a proof see Theorem 2 in Guerre and Sabbah [17]. By standard smoothing theory, we have that (still when $\Delta_{\alpha} \equiv 0$ )

$$
\sup _{\alpha \in A} \sup _{x \in R_{X}}\left|\widetilde{r}_{\alpha}(x)\right|=O_{P}\left(\left(n h^{d}\right)^{-1 / 2} L_{n}\right)
$$

This shows (29) when $\Delta_{\alpha} \equiv 0$. We can move from this case to $\Delta_{\alpha} \neq 0$ by adding to the observations terms of order $O_{P}\left(n^{-1 / 2} h^{-d / 4}\right)$. This changes the local quantiles by at most this amount, and hence (29) still holds when $\Delta_{\alpha} \neq 0$.

In the case of $\widehat{r}_{\alpha}(x)=\widehat{r}_{\alpha, \widehat{\theta}}^{\Delta}(x)+O_{P}\left(n^{-1 / 2-c}\right)$, we have to add to the observations terms of the order $O_{P}\left(L_{n} n^{-1 / 2} h^{-d / 4}\right)=O_{P}\left(\left(n h^{d}\right)^{-1 / 2} L_{n}\right)$. This shows the first statement of the lemma.

Lemma 2. Suppose that the assumptions of Theorem 2 are satisfied. Then,

$$
\sup _{\alpha \in A} \sup _{x \in R_{X}}\left|\widehat{r}_{\alpha}(x)-\widehat{r}_{\alpha}^{\Delta}(x)+\left(\widehat{\theta}(\alpha)-\theta_{0}(\alpha)\right)^{\top} \gamma_{\alpha}(x)\right|=O_{P}\left(n^{-\frac{1}{2}-c}\right) .
$$

Proof. First note that $\widehat{r}_{\alpha}(x)+\left(\widehat{\theta}(\alpha)-\theta_{0}(\alpha)\right)^{\top} \gamma_{\alpha}(x)$ is equal to the quantile estimator we would obtain when we shift all observations $Y_{i}$ in the window around $x$ by the amount $\left(\widehat{\theta}(\alpha)-\theta_{0}(\alpha)\right)^{\top} \gamma_{\alpha}(x)$, and hence we need to show that the distance between this latter estimator ( $\left.\operatorname{say} \widehat{r}_{\alpha, \bmod }(x)\right)$ and $\widehat{r}_{\alpha}^{\Delta}(x)$ is $O_{P}\left(n^{-\frac{1}{2}-c}\right)$ uniformly in $\alpha$ and $x$.

Next, note that if now in addition we perturb all observations in the window around $x$ by adding $m_{\alpha, \widehat{\theta}(\alpha)}\left(X_{i}\right)-m_{\alpha, \theta_{0}(\alpha)}\left(X_{i}\right)-\left(\widehat{\theta}(\alpha)-\theta_{0}(\alpha)\right)^{\top} \gamma_{\alpha}\left(X_{i}\right)$, the quantile estimator $\widehat{r}_{\alpha, \bmod }(x)$ 
will get perturbed by at most the maximal perturbation of the observations, which is of the order $O_{P}\left(n^{-1 / 2-c}\right)$ by Assumption (B4).

After these two perturbations, the quantile estimator is now based on $Y_{i}-m_{\alpha, \theta_{0}(\alpha)}\left(X_{i}\right)+$ $\left(\widehat{\theta}(\alpha)-\theta_{0}(\alpha)\right)^{\top}\left(\gamma_{\alpha}(x)-\gamma_{\alpha}\left(X_{i}\right)\right)$ instead of $Y_{i}-m_{\alpha, \widehat{\theta}(\alpha)}\left(X_{i}\right)$. Finally note that if we apply one more perturbation by subtracting $\left(\widehat{\theta}(\alpha)-\theta_{0}(\alpha)\right)^{\top}\left(\gamma_{\alpha}(x)-\gamma_{\alpha}\left(X_{i}\right)\right)$ for all $X_{i}$ in the window around $x$, the estimator changes by at most $O_{P}\left(h^{-\delta} n^{-1 / 2-\rho} h^{\delta}\right)=O_{P}\left(n^{-1 / 2-\rho}\right)$ by Assumption (B5). The so-obtained estimator equals $\widehat{r}_{\alpha}^{\Delta}(x)$, which shows the statement of the lemma.

Lemma 3. Suppose that the assumptions of Theorem 2 are satisfied. Then,

$$
\sup _{\alpha \in A} \sup _{x \in R_{X}}\left|\widehat{r}_{\alpha}^{\Delta}(x)-\widetilde{r}_{\alpha}^{\Delta}(x)\right|=O_{P}\left(\left(n h^{d}\right)^{-3 / 4} L_{n}\right) .
$$

Proof. Write

$$
\begin{aligned}
& \left|\widehat{r}_{\alpha}^{\Delta}(x)-\widetilde{r}_{\alpha}^{\Delta}(x)\right| \\
& \leq \frac{1}{\inf _{x, \alpha} f_{\varepsilon_{\alpha} \mid X}(0 \mid x)}\left|\sum_{i=1}^{n} W_{n i}(x, h) f_{\varepsilon_{\alpha} \mid X}\left(0 \mid X_{i}\right) \widehat{r}_{\alpha}^{\Delta}(x)+\sum_{i=1}^{n} W_{n i}(x, h)\left(I\left(\varepsilon_{i, \alpha}^{\Delta} \leq 0\right)-\alpha\right)\right| \\
& =\frac{1}{\inf _{x, \alpha} f_{\varepsilon_{\alpha} \mid X}(0 \mid x)}\left|\sum_{i=1}^{n} W_{n i}(x, h) f_{\varepsilon_{\alpha} \mid X}\left(0 \mid X_{i}\right) \widehat{r}_{\alpha}^{\Delta}(x)-\widehat{F}_{\varepsilon_{\alpha}^{\Delta} \mid X}\left(\widehat{r}_{\alpha}^{\Delta}(x) \mid x\right)+\widehat{F}_{\varepsilon_{\alpha}^{\Delta} \mid X}(0 \mid x)\right| \\
& \quad+O_{P}\left(\left(n h^{d}\right)^{-1}\right)
\end{aligned}
$$

where $\widehat{F}_{\varepsilon_{\alpha}^{\Delta} \mid X}(y \mid x)=\sum_{i} W_{n i}(x, h) I\left(\varepsilon_{i, \alpha}^{\Delta} \leq y\right)$. The latter equality follows from the fact that

$$
\begin{aligned}
\left|\widehat{F}_{\varepsilon_{\alpha}^{\Delta} \mid X}\left(\widehat{r}_{\alpha}^{\Delta}(x) \mid x\right)-\alpha\right| & \leq\left|\widehat{F}_{\varepsilon_{\alpha}^{\Delta} \mid X}\left(\widehat{r}_{\alpha}^{\Delta}(x) \mid x\right)-\widehat{F}_{\varepsilon_{\alpha}^{\Delta} \mid X}\left(\widehat{r}_{\alpha}^{\Delta}(x)-\mid x\right)\right| \\
& =O_{P}\left(\left(n h^{d}\right)^{-1}\right) .
\end{aligned}
$$

The following expansion follows from standard kernel smoothing theory, uniformly for $x \in$ $R_{X}, \alpha \in A,|y| \leq a_{n}$ and for sequences $a_{n}$ with $a_{n}^{-1}=O\left(n h^{d}\right)$ :

$$
\begin{aligned}
& \widehat{F}_{\varepsilon_{\alpha}^{\Delta} \mid X}(y \mid x)-\widehat{F}_{\varepsilon_{\alpha}^{\Delta} \mid X}(0 \mid x) \\
& =\sum_{i} W_{n i}(x, h) \int_{0}^{y} f_{\varepsilon_{\alpha} \mid X}\left(u-n^{-1 / 2} h^{-d / 4} \Delta_{\alpha}\left(X_{i}\right) \mid X_{i}\right) d u+O_{P}\left(\left(n h^{d}\right)^{-1 / 2} L_{n} a_{n}^{1 / 2}\right) \\
& \quad=\sum_{i} W_{n i}(x, h) \int_{0}^{y} f_{\varepsilon_{\alpha} \mid X}\left(u \mid X_{i}\right) d u+O_{P}\left(\left(n h^{d}\right)^{-1 / 2} L_{n} a_{n}^{1 / 2}\right)+O_{P}\left(n^{-1 / 2} h^{-d / 4} a_{n}\right) \\
& =y \sum_{i} W_{n i}(x, h) f_{\varepsilon_{\alpha} \mid X}\left(0 \mid X_{i}\right)+O_{P}\left(\left(n h^{d}\right)^{-1 / 2} L_{n} a_{n}^{1 / 2}+a_{n}^{2}\right)+O_{P}\left(n^{-1 / 2} h^{-d / 4} a_{n}\right) .
\end{aligned}
$$


We now apply this bound to $a_{n}=\left(n h^{d}\right)^{-1 / 2} L_{n}$ and $y=\widehat{r}_{\alpha}^{\Delta}(x)$, which is possible thanks to Lemma 1. This combined with (31) shows the statement of the lemma.

For proving Theorem 2, we will make use of the following decomposition, which follows from Lemma 2:

$$
\begin{aligned}
\widehat{T}_{A}= & \int_{A} \int_{R_{X}}\left[\widehat{r}_{\alpha}^{\Delta}(x)-\left(\widehat{\theta}(\alpha)-\theta_{0}(\alpha)\right)^{\top} \gamma_{\alpha}(x)\right]^{2} w(x, \alpha) d x d \alpha+o_{P}\left(n^{-1} h^{-d / 2}\right) \\
= & \int_{A} \int_{R_{X}}\left[\widehat{r}_{\alpha}^{\Delta}(x)^{2}-\bar{r}_{\alpha}^{\Delta}(x)^{2}\right] w(x, \alpha) d x d \alpha \\
& +\int_{A} \int_{R_{X}} E\left\{\bar{r}_{\alpha}^{\Delta}(x)^{2}-\widetilde{r}_{\alpha}^{\Delta}(x)^{2} \mid N(x)\right\} w(x, \alpha) d x d \alpha \\
& +\int_{A} \int_{R_{X}}\left[\bar{r}_{\alpha}^{\Delta}(x)^{2}-\widetilde{r}_{\alpha}^{\Delta}(x)^{2}-E\left\{\bar{r}_{\alpha}^{\Delta}(x)^{2}-\widetilde{r}_{\alpha}^{\Delta}(x)^{2} \mid N(x)\right\}\right] w(x, \alpha) d x d \alpha \\
& -2 \int_{A} \int_{R_{X}}\left[\left(\widehat{r}_{\alpha}^{\Delta}(x)-\widetilde{r}_{\alpha}^{\Delta}(x)\right)\left\{\left(\widehat{\theta}(\alpha)-\theta_{0}(\alpha)\right)^{\top} \gamma_{\alpha}(x)\right\}\right] w(x, \alpha) d x d \alpha \\
& -2 \int_{A} \int_{R_{X}}\left[\widetilde{r}_{\alpha}^{\Delta}(x)\left\{\left(\widehat{\theta}(\alpha)-\theta_{0}(\alpha)\right)^{\top} \gamma_{\alpha}(x)\right\}\right] w(x, \alpha) d x d \alpha \\
& +\int_{A} \int_{R_{X}}\left[\left(\widehat{\theta}(\alpha)-\theta_{0}(\alpha)\right)^{\top} \gamma_{\alpha}(x)\right]^{2} w(x, \alpha) d x d \alpha \\
& +\int_{A} \int_{R_{X}} \widetilde{r}_{\alpha}^{\Delta}(x)^{2} w(x, \alpha) d x d \alpha+o_{P}\left(n^{-1} h^{-d / 2}\right) \\
= & T_{n 1}+\cdots+T_{n 7}+o_{P}\left(n^{-1} h^{-d / 2}\right) .
\end{aligned}
$$

Lemma 4. Suppose that the assumptions of Theorem 2 are satisfied. Then,

$$
T_{n 1}=o_{P}\left(a_{n}\right)
$$

for any sequence $\left\{a_{n}\right\}$ of positive constants tending to zero as $n \rightarrow \infty$.

Proof. Note that

$$
T_{n 1} \leq \sup _{\alpha \in A} \sup _{x \in R_{X}}\left|\widehat{r}_{\alpha}^{\Delta}(x)\right|^{2} \int_{A} \int_{R_{X}} I\left(\left|\widehat{r}_{\alpha}^{\Delta}(x)\right|>L_{n}\left(n h^{d}\right)^{-1 / 2}\right) w(x, \alpha) d x d \alpha .
$$

It is easily seen from Lemma 1 that

$$
\int_{A} \int_{R_{X}} I\left(\left|\widehat{r}_{\alpha}^{\Delta}(x)\right|>L_{n}\left(n h^{d}\right)^{-1 / 2}\right) w(x, \alpha) d x d \alpha=o_{P}\left(a_{n}\right)
$$

for any $a_{n} \rightarrow 0$, since the indicator inside the integral will be zero from some point on. 
From Corollary 1, we get the following result.

Lemma 5. Suppose that the assumptions of Theorem 2 are satisfied. Then,

$$
\sup _{\alpha \in A} \sup _{x \in R_{X}}\left|E\left\{\bar{r}_{\alpha}^{\Delta}(x)^{2}-\widetilde{r}_{\alpha}^{\Delta}(x)^{2} \mid N(x)\right\}\right|=o_{P}\left(\left(n h^{d / 2}\right)^{-1}\right),
$$

and hence, $T_{n 2}=o_{P}\left(\left(n h^{d / 2}\right)^{-1}\right)$.

At this point, we needed the additional assumption $n h^{3 d / 2} / L_{n}^{*} \rightarrow \infty$ for the case that $\Delta_{\alpha} \not \equiv 0$. We now shortly outline what happens if we are on the alternative and if this assumption does not hold. Note that for $\left|m-m_{0}\right|=o\left(m_{0}\right)$

$$
\begin{aligned}
& E\left\{\bar{r}_{\alpha}^{\Delta}(x)^{2}-\widetilde{r}_{\alpha}^{\Delta}(x)^{2} \mid N(x)=m\right\} \\
& \quad=E\left\{\left(\bar{r}_{\alpha}^{\Delta,-}(x)+\Delta_{\alpha}^{h}(x)\right)^{2}-\left(\widetilde{r}_{\alpha}^{\Delta,-}(x)+\Delta_{\alpha}^{h}(x)\right)^{2} \mid N(x)=m\right\} \\
& \quad=E\left\{\bar{r}_{\alpha}^{\Delta,-}(x)^{2}-\widetilde{r}_{\alpha}^{\Delta,-}(x)^{2} \mid N(x)=m\right\}+2 \Delta_{\alpha}^{h}(x) E\left\{\bar{r}_{\alpha}^{\Delta,-}(x) \mid N(x)=m\right\} .
\end{aligned}
$$

For the first term on the right-hand side we get from Corollary 1 that it is of order $o\left(\left(n h^{d / 2}\right)^{-1}\right)$. For $\Delta_{\alpha}^{h}(x)$, one can show that it is equal to $n^{-1 / 2} h^{-d / 4} \Delta_{\alpha}(x)+O\left(L_{n} n^{-1 / 2} h^{-d / 4} h^{2}\right)$. For the term $E\left\{\bar{r}_{\alpha}^{\Delta,-}(x) \mid N(x)=m\right\}$ one can show that it is equal to $\left(n h^{d}\right)^{-1} \rho(x)+O\left(L_{n}\left(n h^{d}\right)^{-3 / 2}\right)$ for some function $\rho$ that does not depend on the function $\Delta_{\alpha}$. This can be done by using the arguments based on Edgeworth expansions that were central in the proof of Theorem 1. This gives that

$$
T_{n 2}=n^{-3 / 2} h^{-5 d / 4} \int_{A} \int_{R_{X}} \Delta_{\alpha}(x) \rho(x) w(x, \alpha) d x d \alpha+o_{P}\left(n^{-3 / 2} h^{-5 d / 4}\right)+o_{P}\left(\left(n h^{d / 2}\right)^{-1}\right) .
$$

Suppose now that $n h^{3 d / 2} \rightarrow 0$. Then it holds that $\left(n h^{d / 2}\right)^{-1}=o\left(n^{-3 / 2} h^{-5 d / 4}\right)$ and using Lemma 10 we get that

$$
\begin{aligned}
T_{n}= & n^{-1} h^{-d} K^{(2)}(0) \int_{A} \alpha(1-\alpha) \int_{R_{X}} \frac{w(x, \alpha)}{f_{X}(x) f_{\varepsilon_{\alpha} \mid X}^{2}(0 \mid x)} d x d \alpha \\
& +n^{-3 / 2} h^{-5 d / 4} \int_{A} \int_{R_{X}} \Delta_{\alpha}(x) \rho(x) w(x, \alpha) d x d \alpha+o_{P}\left(n^{-3 / 2} h^{-5 d / 4}\right) .
\end{aligned}
$$

This implies that the test rejects for large values of $\int_{A} \int_{R_{X}} \Delta_{\alpha}(x) \rho(x) w(x, \alpha) d x d \alpha$. Thus, in this high-dimensional setting the test behaves like a linear test and not like an omnibus test.

Lemma 6. Suppose the assumptions of Theorem 2 are satisfied. Then,

$$
T_{n 3}=O_{P}\left(L_{n} n^{-5 / 4} h^{-3 d / 4}\right)=o_{P}\left(\left(n h^{d / 2}\right)^{-1}\right) .
$$


Proof. For simplicity of exposition of the argument, let us assume that $X_{i}$ is one-dimensional. For arbitrary $j$ and for $k \in\{1,2,3\}$, define

$$
U_{j k}=\int_{A} \int_{I_{j k}}\left[\bar{r}_{\alpha}^{\Delta}(x)^{2}-\widetilde{r}_{\alpha}^{\Delta}(x)^{2}-E\left\{\bar{r}_{\alpha}^{\Delta}(x)^{2}-\widetilde{r}_{\alpha}^{\Delta}(x)^{2} \mid N(x)\right\}\right] w(x, \alpha) d x d \alpha .
$$

Then we can write $T_{n 3}=T_{n 31}+T_{n 32}+T_{n 33}$ with $T_{n 3 k}=\sum_{j} U_{j k}(k=1,2,3)$. The terms $T_{n 31}$, $T_{n 32}$ and $T_{n 33}$ are sums of $O\left(h^{-1}\right)$ conditionally independent summands. The summands are uniformly bounded by a term of order $O_{P}\left(L_{n} n^{-5 / 4} h^{-1 / 4}\right)$. This follows from Lemma 5 , from the fact that $\sup _{\alpha \in A} \sup _{x}\left|\widetilde{r}_{\alpha}^{\Delta}(x)\right|=O_{P}\left(L_{n}(n h)^{-1 / 2}\right)$, see also (30), and from the Bahadur representation for $\bar{r}_{\alpha}^{\Delta}(x)$, given in Lemma 3. It now follows that $T_{n 3 k}=O_{P}\left(L_{n} n^{-5 / 4} h^{-3 / 4}\right)$, which implies the statement of the lemma for $d=1$. For $d>1$ one can use the same approach.

Lemma 7. Suppose the assumptions of Theorem 2 are satisfied. Then,

$$
T_{n 4}=o_{P}\left(\left(n h^{d / 2}\right)^{-1}\right)
$$

Proof. This is obvious, since $T_{n 4}=O_{P}\left(L_{n}\left(n h^{d}\right)^{-3 / 4} n^{-\frac{1}{4}} h^{\frac{d}{4}} / L_{n}^{*}\right)=o_{P}\left(\left(n h^{d / 2}\right)^{-1}\right)$, thanks to Assumption (B5) and Lemma 3.

Lemma 8. Suppose the assumptions of Theorem 2 are satisfied. Then,

$$
T_{n 5}=o_{P}\left(\left(n h^{d / 2}\right)^{-1}\right)
$$

Proof. Write

$$
\begin{aligned}
T_{n 5}= & 2 \int_{A} \int_{R_{X}} \frac{\sum_{i=1}^{n} K\left(\frac{x-X_{i}}{h}\right)\left\{I\left(\varepsilon_{i, \alpha}^{\Delta} \leq 0\right)-\alpha\right\}}{\sum_{i=1}^{n} K\left(\frac{x-X_{i}}{h}\right) f_{\varepsilon_{\alpha} \mid X}\left(0 \mid X_{i}\right)}\left(\widehat{\theta}(\alpha)-\theta_{0}(\alpha)\right)^{\top} \gamma_{\alpha}(x) w(x, \alpha) d x d \alpha \\
= & \frac{2}{n} \int_{A} \int_{R_{X}} \frac{\sum_{i=1}^{n} K\left(\frac{x-X_{i}}{h}\right)\left\{I\left(\varepsilon_{i, \alpha}^{\Delta} \leq 0\right)-\alpha\right\}}{g_{h, \alpha}(x)}\left(\widehat{\theta}(\alpha)-\theta_{0}(\alpha)\right)^{\top} \gamma_{\alpha}(x) w(x, \alpha) d x d \alpha \\
& +o_{P}\left(\left(n h^{d / 2}\right)^{-1}\right) \\
= & 2 \int_{A}\left(\widehat{\theta}(\alpha)-\theta_{0}(\alpha)\right)^{\top} \frac{1}{n} \sum_{i=1}^{n} \rho_{h, \alpha}\left(X_{i}\right)\left\{I\left(\varepsilon_{i, \alpha}^{\Delta} \leq 0\right)-\alpha\right\} d \alpha+o_{P}\left(\left(n h^{d / 2}\right)^{-1}\right),
\end{aligned}
$$

with $g_{h, \alpha}(x)=E\left[K\left(\frac{x-X}{h}\right) f_{\varepsilon_{\alpha} \mid X}(0 \mid X)\right]$ and

$$
\rho_{h, \alpha}(v)=\int_{R_{X}} K\left(\frac{x-v}{h}\right) \frac{\gamma_{\alpha}(x) w(x, \alpha)}{g_{h, \alpha}(x)} d x .
$$


Using the notations $Q_{h, \alpha}\left(X_{i}\right)=\frac{\rho_{h, \alpha}\left(X_{i}\right)}{\sum_{j=1}^{n} \rho_{h, \alpha}\left(X_{j}\right)}, \widehat{F}_{\varepsilon_{\alpha}^{\Delta}}(y)=\sum_{i=1}^{n} Q_{h, \alpha}\left(X_{i}\right) I\left(\varepsilon_{i, \alpha}^{\Delta} \leq y\right)$ and $F_{\varepsilon_{\alpha}^{\Delta}}(y)=P\left(\varepsilon_{\alpha}^{\Delta} \leq y\right)$, we have that

$$
\begin{aligned}
& \frac{1}{n} \sum_{i=1}^{n} \rho_{h, \alpha}\left(X_{i}\right)\left\{I\left(\varepsilon_{i, \alpha}^{\Delta} \leq 0\right)-\alpha\right\} \\
& \quad=\left[\widehat{F}_{\varepsilon_{\alpha}^{\Delta}}(0)-\alpha\right]\left(\frac{1}{n} \sum_{i=1}^{n} \rho_{h, \alpha}\left(X_{i}\right)\right) \\
& \quad=\left[\widehat{F}_{\varepsilon_{\alpha}^{\Delta}}(0)-F_{\varepsilon_{\alpha}^{\Delta}}(0)\right]\left(\frac{1}{n} \sum_{i=1}^{n} \rho_{h, \alpha}\left(X_{i}\right)\right)+\left[F_{\varepsilon_{\alpha}^{\Delta}}(0)-\alpha\right]\left(\frac{1}{n} \sum_{i=1}^{n} \rho_{h, \alpha}\left(X_{i}\right)\right) \\
& \quad=O_{P}\left(n^{-1 / 2}\right)+O_{P}\left(n^{-1 / 2} h^{-d / 4}\right)
\end{aligned}
$$

uniformly in $\alpha \in A$, and hence the statement of the lemma holds because of (32) and (B5).

Lemma 9. Suppose the assumptions of Theorem 2 are satisfied. Then,

$$
T_{n 6}=o_{P}\left(\left(n h^{d / 2}\right)^{-1}\right)
$$

Proof. The statement of the lemma follows from (B5).

Lemma 10. Suppose the assumptions of Theorem 2 are satisfied. Then,

$$
n h^{d / 2} T_{n 7}-b_{h, A} \stackrel{d}{\rightarrow} N\left(D_{A}, V_{A}\right)
$$

Proof. The proof is very similar to the proof of for example, Proposition 1 in Härdle and Mammen [19]. Write

$$
\begin{aligned}
T_{n 7}= & n^{-2} \sum_{i, j} \int_{A} \int_{R_{X}} K\left(\frac{x-X_{i}}{h}\right) K\left(\frac{x-X_{j}}{h}\right)\left\{I\left(\varepsilon_{i, \alpha}^{\Delta} \leq 0\right)-\alpha\right\}\left\{I\left(\varepsilon_{j, \alpha}^{\Delta} \leq 0\right)-\alpha\right\} \\
& \times \widehat{g}_{\alpha}(x)^{-2} w(x, \alpha) d x d \alpha,
\end{aligned}
$$

where $\widehat{g}_{\alpha}(x)=n^{-1} \sum_{i=1}^{n} K\left(\frac{x-X_{i}}{h}\right) f_{\varepsilon_{\alpha} \mid X}\left(0 \mid X_{i}\right)$. By writing $I\left(\varepsilon_{i, \alpha}^{\Delta} \leq 0\right)-\alpha=\left[I\left(\varepsilon_{i, \alpha}^{\Delta} \leq 0\right)-\right.$ $\left.I\left(\varepsilon_{i, \alpha} \leq 0\right)\right]+\left[I\left(\varepsilon_{i, \alpha} \leq 0\right)-\alpha\right]$, we can decompose $T_{n 7}$ into $T_{n 7}=T_{n 71}+T_{n 72}+2 T_{n 73}$. As in Härdle and Mammen [19], $T_{n 73}$ is negligible. Straightforward calculations show that $T_{n 71}=$ $\left(n h^{d / 2}\right)^{-1}\left(D_{A}+o_{P}(1)\right)$. Indeed,

$$
\begin{aligned}
E & \left(T_{n 71} \mid X_{1}, \ldots, X_{n}\right) \\
& =n^{-2} \sum_{i, j} \int_{A} \int_{R_{X}} K\left(\frac{x-X_{i}}{h}\right) K\left(\frac{x-X_{j}}{h}\right)
\end{aligned}
$$




$$
\begin{aligned}
& \times\left[F_{\varepsilon_{\alpha} \mid X}\left(-n^{-1 / 2} h^{-d / 4} \Delta_{\alpha}\left(X_{i}\right) \mid X_{i}\right)-F_{\varepsilon_{\alpha} \mid X}\left(0 \mid X_{i}\right)\right] \\
& \times\left[F_{\varepsilon_{\alpha} \mid X}\left(-n^{-1 / 2} h^{-d / 4} \Delta_{\alpha}\left(X_{j}\right) \mid X_{j}\right)-F_{\varepsilon_{\alpha} \mid X}\left(0 \mid X_{j}\right)\right] \widehat{g}_{\alpha}(x)^{-2} w(x, \alpha) d x d \alpha \\
= & n^{-2} \sum_{i, j} \int_{A} \int_{R_{X}} K\left(\frac{x-X_{i}}{h}\right) K\left(\frac{x-X_{j}}{h}\right) f_{\varepsilon_{\alpha} \mid X}\left(0 \mid X_{i}\right) f_{\varepsilon_{\alpha} \mid X}\left(0 \mid X_{j}\right) \\
& \times n^{-1} h^{-d / 2} \Delta_{\alpha}\left(X_{i}\right) \Delta_{\alpha}\left(X_{j}\right) \widehat{g}_{\alpha}(x)^{-2} w(x, \alpha) d x d \alpha\left(1+o_{P}(1)\right) \\
= & n^{-1} h^{-d / 2} \int_{A} \int_{R_{X}} \Delta_{\alpha}^{2}(x) w(x, \alpha) d x d \alpha\left(1+o_{P}(1)\right) \\
= & n^{-1} h^{-d / 2} D_{A}\left(1+o_{P}(1)\right) .
\end{aligned}
$$

Next, write $T_{n 72}=T_{n 72 a}+T_{n 72 b}$ with

$$
\begin{aligned}
& T_{n 72 a}=\frac{1}{n^{2}} \sum_{i=1}^{n} U_{n i i}, \\
& T_{n 72 b}=\frac{1}{n^{2}} \sum_{i \neq j} U_{n i j},
\end{aligned}
$$

where

$$
\begin{aligned}
U_{n i j}= & \int_{A} \int_{R_{X}} K\left(\frac{x-X_{i}}{h}\right) K\left(\frac{x-X_{j}}{h}\right)\left\{I\left(\varepsilon_{i, \alpha} \leq 0\right)-\alpha\right\}\left\{I\left(\varepsilon_{j, \alpha} \leq 0\right)-\alpha\right\} \\
& \times \widehat{g}_{\alpha}(x)^{-2} w(x, \alpha) d x d \alpha .
\end{aligned}
$$

By calculating its mean and variance it can be checked that $n h^{d / 2} T_{n 72 a}=b_{h, A}+o_{P}(1)$. Thus for the lemma it remains to check that $n h^{d / 2} T_{n 72 b} \stackrel{d}{\rightarrow} N\left(0, V_{A}\right)$. For the proof of this claim one can proceed as in Härdle and Mammen [19] and apply the central limit theorem for U-statistics of de Jong [9]. For this purpose, one has to verify that $n^{2} h^{d} \operatorname{Var}\left(T_{n 72 b}\right) \rightarrow V_{A}$, $\max _{1 \leq i \leq n} \sum_{j=1}^{n} \operatorname{Var}\left(U_{n i j}\right) / \operatorname{Var}\left(T_{n 72 b}\right) \rightarrow 0$ and $E\left[T_{n 72 b}^{4}\right] /\left(\operatorname{Var}\left(T_{n 72 b}\right)\right)^{2} \rightarrow 3$. This can be done by straightforward but tedious calculations.

Proof of Theorem 2. The theorem follows immediately from Lemmas 4-10. Lemmas 4-9 imply the negligibility of the terms $T_{n 1}, \ldots, T_{n 6}$. Lemma 10 shows the asymptotic normality of $n h^{d / 2} T_{n 7}$.

\section{Proof of Theorem 3}

The theorem can be shown by verification of the conditions of the central limit theorem for Ustatistics of de Jong [9], in the same way as was done in the proof of Lemma 10. The crucial point in the proof is to note that $I\left(U_{i} \leq \alpha\right)$ has the same distribution as $I\left(\varepsilon_{i, \alpha} \leq 0\right)$, and hence the calculations in the proof of Lemma 10 go through in this proof. 


\section{Acknowledgments}

Research of the first author was prepared within the framework of a subsidy granted to the HSE by the Government of the Russian Federation for the implementation of the Global Competitiveness Program and it was supported by Deutsche Forschungsgemeinschaft through the Research Training Group RTG 1953. The research of the second author was supported by the European Research Council (2016-2021, Horizon 2020 / ERC grant agreement No. 694409), and by IAP research network grant nr. P7/06 of the Belgian government (Belgian Science Policy).

\section{References}

[1] Aït-Sahalia, Y., Fan, J. and Peng, H. (2009). Nonparametric transition-based tests for jump diffusions. J. Amer. Statist. Assoc. 104 1102-1116. MR2750239

[2] Angrist, J., Chernozhukov, V. and Fernández-Val, I. (2006). Quantile regression under misspecification, with an application to the U.S. wage structure. Econometrica 74 539-563. MR2207400

[3] Bhattacharya, R.N. and Ranga Rao, R. (1976). Normal Approximation and Asymptotic Expansions. Wiley Series in Probability and Mathematical Statistics Wiley, New York. MR0436272

[4] Bierens, H.J. and Ginther, D. (2001). Integrated conditional moment testing of quantile regression models. Empir. Econ. 26 307-324.

[5] Chao, S.-K., Volgushev, S. and Cheng, G. (2017). Quantile processes for semi and nonparametric regression. Electron. J. Stat. 11 3272-3331. MR3708539

[6] Chaudhuri, P. (1991). Nonparametric estimates of regression quantiles and their local Bahadur representation. Ann. Statist. 19 760-777. MR1105843

[7] Conde-Amboage, M., Sánchez-Sellero, C. and González-Manteiga, W. (2015). A lack-of-fit test for quantile regression models with high-dimensional covariates. Comput. Statist. Data Anal. 88 128-138. MR3332022

[8] De Backer, M., El Ghouch, A. and Van Keilegom, I. (2017). Semiparametric copula quantile regression for complete or censored data. Electron. J. Stat. 11 1660-1698. MR3639560

[9] de Jong, P. (1987). A central limit theorem for generalized quadratic forms. Probab. Theory Related Fields 75 261-277. MR0885466

[10] Dette, H. and Spreckelsen, I. (2004). Some comments on specification tests in nonparametric absolutely regular processes. J. Time Series Anal. 25 159-172. MR2045571

[11] El Ghouch, A. and Van Keilegom, I. (2009). Local linear quantile regression with dependent censored data. Statist. Sinica 19 1621-1640. MR2589201

[12] Engel, E. (1857). Die vorherrschenden Gewerbszweige in den Gerichtsämtern mit Beziehung auf die Productions- und Consumtionsverhältnisse des Königreichs Sachsen. II. Das Gesetz der Dichtigkeit. Zeitschrift des statistischen Bureaus des Königlich Sächsischen Ministerium des Inneren 3 8-9 153182.

[13] Fan, J., Zhang, C. and Zhang, J. (2001). Generalized likelihood ratio statistics and Wilks phenomenon. Ann. Statist. 29 153-193. MR1833962

[14] Gao, J. and Hong, Y. (2008). Central limit theorems for generalized $U$-statistics with applications in nonparametric specification. J. Nonparametr. Stat. 20 61-76. MR2396276

[15] González Manteiga, W. and Cao, R. (1993). Testing the hypothesis of a general linear model using nonparametric regression estimation. TEST 2 161-188. MR1265489

[16] Guerre, E. and Lavergne, P. (2002). Optimal minimax rates for nonparametric specification testing in regression models. Econometric Theory 18 1139-1171. MR1926017 
[17] Guerre, E. and Sabbah, C. (2012). Uniform bias study and Bahadur representation for local polynomial estimators of the conditional quantile function. Econometric Theory 28 87-129. MR2899215

[18] Haag, B.R. (2008). Non-parametric regression tests using dimension reduction techniques. Scand. J. Stat. 35 719-738. MR2468872

[19] Härdle, W. and Mammen, E. (1993). Comparing nonparametric versus parametric regression fits. Ann. Statist. 21 1926-1947. MR1245774

[20] He, X. and Ng, P. (1999). Quantile splines with several covariates. J. Statist. Plann. Inference 75 343-352. The Seventh Eugene Lukacs Conference (Bowling Green, OH, 1997). MR1678981

[21] He, X., Ng, P. and Portnoy, S. (1998). Bivariate quantile smoothing splines. J. R. Stat. Soc. Ser. B. Stat. Methodol. 60 537-550. MR1625950

[22] He, X. and Zhu, L.-X. (2003). A lack-of-fit test for quantile regression. J. Amer. Statist. Assoc. 98 1013-1022. MR2041489

[23] Hjellvik, V., Yao, Q. and Tjøstheim, D. (1998). Linearity testing using local polynomial approximation. J. Statist. Plann. Inference 68 295-321. MR1629587

[24] Hoderlein, S. and Mammen, E. (2009). Identification and estimation of local average derivatives in non-separable models without monotonicity. Econom. J. 12 1-25. MR2500194

[25] Hong, S.-Y. (2003). Bahadur representation and its applications for local polynomial estimates in nonparametric $M$-regression. J. Nonparametr. Stat. 15 237-251. MR1981463

[26] Horowitz, J.L. and Spokoiny, V.G. (2002). An adaptive, rate-optimal test of linearity for median regression models. J. Amer. Statist. Assoc. 97 822-835. MR1941412

[27] Ingster, Yu.I. (1993). Asymptotically minimax hypothesis testing for nonparametric alternatives. I. Math. Methods Statist. 2 85-114. MR1257978

[28] Ingster, Yu.I. (1993). Asymptotically minimax hypothesis testing for nonparametric alternatives. II. Math. Methods Statist. 2 171-189. MR1257983

[29] Ingster, Yu.I. (1993). Asymptotically minimax hypothesis testing for nonparametric alternatives. III. Math. Methods Statist. 2 249-268. MR1259685

[30] Koenker, R. (2005). Quantile Regression. Econometric Society Monographs 38. Cambridge Univ. Press, Cambridge. MR2268657

[31] Koenker, R. and Machado, J.A.F. (1999). Goodness of fit and related inference processes for quantile regression. J. Amer. Statist. Assoc. 94 1296-1310. MR1731491

[32] Koenker, R. and Xiao, Z. (2002). Inference on the quantile regression process. Econometrica $701583-$ 1612. MR1929979

[33] Kong, E., Linton, O. and Xia, Y. (2010). Uniform Bahadur representation for local polynomial estimates of $M$-regression and its application to the additive model. Econometric Theory 26 1529-1564. MR2684794

[34] Kreiss, J.-P., Neumann, M.H. and Yao, Q. (2008). Bootstrap tests for simple structures in nonparametric times series regression. Stat. Interface 1367-380. MR2476752

[35] Lee, Y.K. and Lee, E.R. (2008). Kernel methods for estimating derivatives of conditional quantiles. J. Korean Statist. Soc. 37 365-373. MR2467903

[36] Leucht, A. (2012). Degenerate $U$ - and $V$-statistics under weak dependence: Asymptotic theory and bootstrap consistency. Bernoulli 18 552-585. MR2922461

[37] Li, Q. and Racine, J.S. (2008). Nonparametric estimation of conditional CDF and quantile functions with mixed categorical and continuous data. J. Bus. Econom. Statist. 26 423-434. MR2459343

[38] Rothe, C. and Wied, D. (2013). Misspecification testing in a class of conditional distributional models. J. Amer. Statist. Assoc. 108 314-324. MR3174622

[39] Su, L. and White, H.L. (2012). Conditional independence specification testing for dependent processes with local polynomial quantile regression. In Essays in Honor of Jerry Hausman. Adv. Econom. 29 355-434. Emerald, Bingley. MR3494865 
[40] Truong, Y.K. (1989). Asymptotic properties of kernel estimators based on local medians. Ann. Statist. 17 606-617. MR0994253

[41] Volgushev, S., Birke, M., Dette, H. and Neumeyer, N. (2013). Significance testing in quantile regression. Electron. J. Stat. 7 105-145. MR3020416

[42] Volgushev, S., Chao, S. and Cheng, G. (2017). Distributed inference for quantile regression processes. Preprint. Available at arXiv:1701.060088v3.

[43] Yu, K. and Jones, M.C. (1998). Local linear quantile regression. J. Amer. Statist. Assoc. 93 228-237. MR1614628

[44] Zheng, J.X. (1996). A consistent test of functional form via nonparametric estimation techniques. J. Econometrics 75 263-289. MR1413644

[45] Zheng, J.X. (1998). A consistent nonparametric test of parametric regression models under conditional quantile restrictions. Econometric Theory 14 123-138. MR1613710

Received December 2015 and revised May 2017 